\title{
The Reactor Antineutrino Anomaly
}

\author{
G. Mention, ${ }^{1}$ M. Fechner, ${ }^{1}$ Th. Lasserre, ${ }^{1,2, \circledast ~ T h . ~ A . ~ M u e l l e r, ~}{ }^{3}$ D. Lhuillier, ${ }^{3}$ M. Cribier,${ }^{1,2}$ and A. Letourneau ${ }^{3}$ \\ ${ }^{1}$ CEA, Irfu, SPP, Centre de Saclay, F-91191 Gif-sur-Yvette, France \\ ${ }^{2}$ Astroparticule et Cosmologie APC, 10 rue Alice Domon et Léonie Duquet, 75205 Paris cedex 13, France \\ ${ }^{3}$ CEA, Irfu, SPhN, Centre de Saclay, F-91191 Gif-sur-Yvette, France
}

(Dated: March 24, 2011)

\begin{abstract}
Recently, new reactor antineutrino spectra have been provided for ${ }^{235} \mathrm{U},{ }^{239} \mathrm{Pu},{ }^{241} \mathrm{Pu}$, and ${ }^{238} \mathrm{U}$, increasing the mean flux by about 3 percent. To a good approximation, this reevaluation applies to all reactor neutrino experiments. The synthesis of published experiments at reactor-detector distances $<100 \mathrm{~m}$ leads to a ratio of observed event rate to predicted rate of $0.976 \pm 0.024$. With our new flux evaluation, this ratio shifts to $0.943 \pm 0.023$, leading to a deviation from unity at $98.6 \%$ C.L. which we call the reactor antineutrino anomaly. The compatibility of our results with the existence of a fourth non-standard neutrino state driving neutrino oscillations at short distances is discussed. The combined analysis of reactor data, gallium solar neutrino calibration experiments, and MiniBooNE$\nu$ data disfavors the no-oscillation hypothesis at $99.8 \%$ C.L. The oscillation parameters are such that $\left|\Delta m_{\text {new }}^{2}\right|>1.5 \mathrm{eV}^{2}(95 \%)$ and $\sin ^{2}\left(2 \theta_{\text {new }}\right)=0.14 \pm 0.08(95 \%)$. Constraints on the $\theta_{13}$ neutrino mixing angle are revised.
\end{abstract}

\section{INTRODUCTION}

Neutrino oscillation experiments over the last twenty years have established a picture of neutrino mixing and masses that explains the results of solar, atmospheric and reactor neutrino experiments [1]. These experiments are consistent with the mixing of $\nu_{e}, \nu_{\mu}$ and $\nu_{\tau}$ with three mass eigenstates, $\nu_{1}, \nu_{2}$ and $\nu_{3}$. In particular, the mass differences are required to be $\left|\Delta m_{31}^{2}\right| \simeq 2.410^{-3} \mathrm{eV}^{2}$ and $\Delta m_{21}^{2} /\left|\Delta m_{31}^{2}\right| \simeq 0.032$.

Reactor experiments have played an important role in the establishment of this pattern. In experiments at distances $<100 \mathrm{~m}$ from the reactor core, at ILL-Grenoble, Goesgen, Rovno, Krasnoyarsk, Savannah River and Bugey [2 9, 11], the measured rate of $\bar{\nu}_{e}$ was found to be in reasonable agreement with that predicted from the reactor antineutrino spectra, though slightly lower than expected, with the measured/expected ratio at $0.976 \pm 0.024$ (including recent revisions of the neutron mean lifetime). This opened the way to longer baseline experiments measuring the $\bar{\nu}_{e}$ survival probability $P_{e e}$ at various distances.

Including the three known active neutrinos, $P_{e e}$ can be written in first approximation as

$$
P_{e e}^{2 \nu}(L)=1-\sin ^{2}\left(2 \theta_{i}\right) \sin ^{2}\left(1.27 \frac{\Delta m_{i}^{2}\left[\mathrm{eV}^{2}\right] L[\mathrm{~m}]}{E_{\bar{\nu}_{e}}[\mathrm{MeV}]}\right),
$$

where $\Delta m_{i}^{2}=\Delta m_{21}^{2}$ and $\theta_{i} \sim \theta_{12}$ if the baseline exceeds a few tens of kilometers, and $\Delta m_{i}^{2}=\Delta m_{31}^{2}$ and $\theta_{i}=\theta_{13}$ if it does not exceed a few kilometers [12].

The CHOOZ experiment was located $1 \mathrm{~km}$ away from two $4.3 \mathrm{GW}$ reactors [13], and did not report any neutrino oscillation in the parameter region $\Delta m_{31}^{2}>10^{-3} \mathrm{eV}^{2}$. In addition CHOOZ data provides the world's best constraint on the $\theta_{13}$ mixing angle value, at $\sin ^{2}\left(2 \theta_{13}\right)<0.14$

\footnotetext{
* Corresponding author: thierry.lasserre@cea.fr
}

(90\% C.L.) for $\left|\Delta m_{31}^{2}\right| \simeq 2.410^{-3} \mathrm{eV}^{2}$. Forthcoming kilometer baseline experiments with multiple detectors are underway to provide a clean measurement of the last undetermined neutrino mixing angle $\theta_{13}$ [14 16$]$.

The KamLAND experiment detected electron antineutrinos from surrounding reactors located at an average distance of $180 \mathrm{~km}$, thus probing the $\Delta m_{21}^{2} \sim 10^{-4} \mathrm{eV}^{2}$ region. KamLAND successfully reported a strong oscillation signal in agreement with solar neutrino data [17]. A three-flavor oscillation treatment shows that KamLAND also has a weak sensitivity to the $\theta_{13}$ mixing angle, as $P_{e e} \sim \cos ^{4} \theta_{13} P_{e e}^{2 \nu}$, leading to the anti-correlation of $\sin ^{2} \theta_{13}$ and $\sin ^{2} \theta_{12}$. Recent global fits including solar neutrino and reactor oscillation data indicated a preference for non-zero $\theta_{13}$ at about $1.5 \sigma$ [18, 19].

In preparation for the Double Chooz reactor experiment, we have re-evaluated the specific reactor antineutrino flux ( $\nu /$ fission), improving the electron to antineutrino data conversion [20]. The method relies on detailed knowledge of the decays of thousands of fission products, while the previous conversion procedure used a phenomenological model based on 30 effective beta branches. Both methods are constrained by the well-measured ILL spectrum of fission induced electrons that accompanies the antineutrinos $[21-24]$.

The new calculation results in a small increase in the flux of $3.5 \%$. Although this increase has no significant effect on KamLAND's solar parameter results, when combined with the previously reported small deficits at nearer distances, it results in a larger average deficit of $5.7 \%$, at $0.943 \pm 0.023$. We call it the reactor antineutrino anomaly, significant at the level of $98.6 \%$ C.L. This deficit is consistent with being independent from the distance to the reactor core at distances $\gtrsim 15$ meters, the distance of the Bugey-3 experiment [4].

If this deficit is due to neutrino mixing, it could be explained by an energy-independent suppression of the $\bar{\nu}_{e}$ flux at distances $\gtrsim 15$ meters. This requires a neutrino with a $\left|\Delta m_{\text {new }}^{2}\right| \gtrsim 1 \mathrm{eV}^{2}$. The mixing amplitude with the 
$\nu_{e}$ must be $\sin ^{2}\left(2 \theta_{\text {new }}\right) \sim 0.115$. The required $\left|\Delta m_{\text {new }}^{2}\right|$ is significantly larger than those required by solar and atmospheric experiments. This suggests, if the neutrino mixing hypothesis is the correct explanation, the existence of a fourth neutrino, beyond the standard model. We would like to stress here that other explanations are also possible, such as a correlated artifact in the experiments, or an erroneous prediction of the antineutrino flux from nuclear reactor cores.

We begin by discussing the use of new antineutrino spectra and provide a revised value of the predicted cross section per fission, in Section [II We then revisit the short baseline oscillation results $(<100 \mathrm{~m})$ and $\mathrm{CHOOZ}$ in Section III revealing a reactor antineutrino anomaly, which is discussed in Section IV] The compatibility of our results with the existence of a fourth non-standard neutrino state is discussed in Section $\mathrm{V}$. The CHOOZ and KamLAND sensitivities to the $\theta_{13}$ mixing angle are revisited in Sections $\mathrm{VIA}$ and VIB Their combination is discussed in Section VIC Two experimental programs for testing the anomaly are discussed in Section VII. We conclude in Section VIII,

\section{NEW PREDICTED CROSS SECTION PER FISSION}

Fission reactors release about $10^{20} \bar{\nu}_{e} \mathrm{GW}^{-1} \mathrm{~s}^{-1}$, which mainly come from the beta decays of the fission products of ${ }^{235} \mathrm{U},{ }^{238} \mathrm{U},{ }^{239} \mathrm{Pu}$, and ${ }^{241} \mathrm{Pu}$. The emitted antineutrino spectrum is then given by:

$$
S_{\text {tot }}\left(E_{\nu}\right)=\sum_{k} f_{k} S_{k}\left(E_{\nu}\right),
$$

where $f_{k}$ refers to the contribution of the main fissile nuclei to the total number of fissions of the $\mathrm{k}^{\text {th }}$ branch, and $S_{k}$ to their corresponding neutrino spectrum per fission.

For the last 25 years the $\bar{\nu}_{e}$ spectra have been estimated from measurements of the total electron spectra associated with the beta decays of all fission products of ${ }^{235} \mathrm{U},{ }^{239} \mathrm{Pu}$, and ${ }^{241} \mathrm{Pu}$. Thin target foils of these isotopes were irradiated with thermal neutrons at the ILL reactor $[22-24]$. The measured spectra then had to be converted from electron to antineutrino spectra invoking a set of 30 effective beta-branches, adjusted to reproduce the total electron spectrum [39].

Recently we revisited the conversion procedure with a novel mixed-approach combining the accurate reference of the ILL electron spectra with the physical distribution of beta branches of all fission products provided by the nuclear databases [20]. This new approach provided a better handle on the systematic errors of the conversion. Although it did not reduce the final error budget, it led to a systematic shift of about $3 \%$ in the normalization of ${ }^{235} \mathrm{U},{ }^{239} \mathrm{Pu}$, and ${ }^{241} \mathrm{Pu}$ antineutrino fluxes, respectively. This normalization shift has been attributed to two main systematic effects in the original conversion of the ILL electron data. At low energy $\left(E_{\nu}<4 \mathrm{MeV}\right)$ the implementation of Coulomb and weak magnetism corrections to the Fermi theory in the new approach turned out to deviate from the effective linear correction $\left(0.65 \times\left(E_{\nu}-4 \mathrm{MeV}\right)\right.$ in \%) used in the previous work. At high energy $\left(E_{\nu}>4 \mathrm{MeV}\right)$, the converted antineutrino spectra become very sensitive to the knowledge of the charge $Z$ of the nuclei contributing to the total spectrum. In the previous approach, only the mean dependence of $Z$ versus the end-point of the effective betabranches had been used while in the new conversion we had access to the complete distribution, nucleus by nucleus. These two effects could be numerically studied and confirmed on various independent sets of beta-branches.

Because ${ }^{238} \mathrm{U}$ nuclei undergo fission with fast neutrons, the associated electron spectrum could not be measured in the thermal neutron flux of the ILL reactor. Therefore the $a b$ initio summation of the $\bar{\nu}_{e}$ from all possible beta decays of fission products was performed to predict the neutrino spectrum [25]. In Ref. [20] we provided a new prediction with an estimated relative uncertainty of the order of $15 \%$ in the $2-8 \mathrm{MeV}$ range. This uncertainty of $a b$ initio calculations is still too large to be generalized to all isotopes but it is sufficiently accurate in the case of ${ }^{238} \mathrm{U}$, which contributes to less than $10 \%$ of the total fission rate for all reactors considered in this work. An ongoing measurement at the FRM II reactor in Garching will soon provide experimental constraints [26].

When predicting the antineutrino spectrum of a reactor $S_{\text {tot }}\left(E_{\nu}\right)$, a weighted sum of the four antineutrino spectra $S_{k}\left(E_{\nu}\right)$ is computed according to the considered fuel composition, which can be different in each experiment. The object of this article is to analyze the impact of the above-mentioned $\sim 3$ percent shift on past, present, and future experiments.

Generally reactor neutrino oscillation experiments search for the reaction:

$$
\bar{\nu}_{e}+p \rightarrow e^{+}+n,
$$

where an electron antineutrino interacts with a free proton in a detector, often filled with scintillator. The reaction cross section can be precisely computed with the V-A theory of weak interaction [29]:

$$
\sigma_{\mathrm{V}-\mathrm{A}}\left(E_{e}\right)=\kappa p_{e} E_{e}\left(1+\delta_{\mathrm{rec}}+\delta_{\mathrm{wm}}+\delta_{\mathrm{rad}}\right),
$$

$p_{e}$ and $E_{e}$ being the momentum and energy of the positron, and the $\delta$ being the energy dependent recoil $\left(\delta_{\text {rec }}\right)$, weak magnetism $\left(\delta_{\mathrm{wm}}\right)$, and radiative $\left(\delta_{\text {rad }}\right)$ corrections. On the one hand, the prefactor $\kappa$ can be written

$$
\kappa=\frac{G_{F}^{2} \cos ^{2} \theta_{C}}{\pi}\left(1+\Delta_{\text {inner }}^{R}\right)\left(1+3 \lambda^{2}\right),
$$

where $G_{F}$ is the Fermi constant, $\theta_{C}$ the Cabibbo angle, $\Delta_{\text {inner }}^{R}=0.024$ the inner radiative corrections taken from Ref. [28], and $\lambda=1.2694$ the form factor ratio of the axial to vector coupling constant. Parameters are taken by default to their latest PDG average values [1]. Using this parameterization Ref. [30] obtained a reference value 
of $\kappa=0.95210^{-43} \mathrm{~cm}^{2}$, using $\lambda=1.2670$ close to the average value of the year 1999 [31]. This value must now be updated to 1.2694 [1], leading to $\kappa=0.95510^{-43} \mathrm{~cm}^{2}$.

On the other hand, Eq. 4 can be normalized to the $\beta$-decay of the free neutrons and $\kappa$ can be written as:

$$
\kappa=\frac{2 \pi^{2}}{m_{e}^{5} f^{R} \tau_{n}}
$$

$\tau_{n}$ being the neutron mean lifetime, and $f^{R}=$ $1.71465(15)$ the phase-space factor for beta-decay of the free neutron taken from Ref. [27], including outer radiative corrections. Over the last 15 years the neutron mean lifetime has evolved, from $926 \mathrm{~s}$ (value used in the ILL experiment 2]) to its current PDG average value of $885.7 \mathrm{~s}$ [1]. Based on this parameterization the current prefactor $\kappa$ is $0.95610^{-43} \mathrm{~cm}^{2}$. This is our default value in this publication. For the computation of the differential cross section we used [29]. Our results agree within $0.1 \%$ with the results published in Ref. 30] that supersede those of Ref. [39].

It is worth mentioning that there is an ongoing controversy about the world average neutron lifetime, with Ref. 34] finding $\tau_{n}=878 \mathrm{~s}$, and making the world average 'suspect'. A lower value has also been found by the MAMBO-2 group [35]. The new world average should then evolve and settle to 881.4(1.4) s in 2011 (Ref. 35] and private communication from K. Schreckenbach).

We note here that the average value of $\lambda$ may depend on neutron lifetime measurements. However other experiments studying angular correlations between the neutron spin and the emitted electron provide independent measurements of $\lambda$. Recently Ref. 32 finds 1.27590 and Ref. 33 favors $\lambda=1.2750(9)$. A value of $\lambda=1.274$ would be consistent with the latest neutron life time measurements, to be averaged to $\tau_{n}=881.4 \mathrm{~s}$ [35]. Thus the latest values of $\lambda$ and $\tau_{n}$ point to a reevaluation of the prefactor $\kappa$ to $0.96110^{-43} \mathrm{~cm}^{2}$. With this change, the cross section would increase by $0.5 \%$ compared to the calculations in this work.

The outgoing positron and incoming antineutrino energies are related by

$$
E_{\nu}=E_{e}+\Delta+\frac{E_{e}\left(E_{e}+\Delta\right)}{M}+\frac{1}{2} \frac{\left(\Delta^{2}-m_{e}^{2}\right)}{M},
$$

where $\Delta=M_{n}-M_{p}[29,39]$. The prediction of the cross section per fission is defined as:

$$
\sigma_{f}^{\text {pred }}=\int_{0}^{\infty} S_{\text {tot }}\left(E_{\nu}\right) \sigma_{\mathrm{V}-\mathrm{A}}\left(E_{\nu}\right) d E_{\nu}=\sum_{k} f_{k} \sigma_{f, k}^{\text {pred }}
$$

where $S_{\text {tot }}$ is the model dependent reactor neutrino spectrum for a given average fuel composition $\left(f_{k}\right)$ and $\sigma_{\mathrm{V}-\mathrm{A}}$ is the theoretical cross section of reaction (3). The $\sigma_{f, k}^{\text {pred }}$ are the predicted cross sections for each fissile isotope. Experiments at baselines below $100 \mathrm{~m}$ reported either the ratios of the measured to predicted cross section per fission, or the ratios $(\mathrm{R})$ of the observed event rate to the predicted rate.
Accounting for new reactor antineutrino spectra [20] the normalization of predicted antineutrino rates, $\sigma_{f, k}^{\mathrm{pred}}$, is shifted by $+2.5 \%,+3.1 \%,+3.7 \%,+9.8 \%$ for $\mathrm{k}={ }^{235} \mathrm{U}$, ${ }^{239} \mathrm{Pu},{ }^{241} \mathrm{Pu}$, and ${ }^{238} \mathrm{U}$ respectively (see Table I). In the case of ${ }^{238} \mathrm{U}$ the completeness of nuclear databases over the years largely explains the $+9.8 \%$ shift from the reference computations [25]. The new predicted cross section for any fuel composition can be computed from Eq. (8). By default our new computation takes into account the so-called off-equilibrium correction of the antineutrino fluxes (increase in fluxes caused by the decay of long-lived fission products).

\section{IMPACT ON PAST EXPERIMENTAL RESULTS}

In the eighties and nineties, experiments were performed at a few tens of meters from nuclear reactor cores at ILL, Goesgen, Rovno, Krasnoyarsk, Bugey (so called 3 and 4) and Savannah River [2 9, 11]. Following these pioneering results middle- and long-baseline experiments were performed at CHOOZ [13] and KamLAND [17] in the late nineties and after. In this section we revised each ratio of observed event rate to predicted rate. The observed event rates and their associated errors are unchanged. The predicted rates are reevaluated separately in each experimental case.

\section{A. The Bugey-4 integral measurement}

The Bugey-4 experiment [3] performed the most precise measurement of the inverse beta decay cross section, obtaining $\sigma_{f}^{\text {Bugey }}=5.752 \pm 0.081$ in units of $10^{-43} \mathrm{~cm}^{2} /$ fission. This experimental result was compared to the predicted cross section per fission, $\sigma_{f}^{\text {pred,old }}$.

Using Ref. 3] inputs, and the former converted neutrino spectra from 22, 40, 41] (except for the ${ }^{238} \mathrm{U}$ neutrino spectrum taken from [20]), we get $\sigma_{f}^{\text {pred,old }}=5.85010^{-43} \mathrm{~cm}^{2} /$ fission, in good agreement with Bugey-4's estimation (see Table I). We used the average fuel composition of Ref. 3], ${ }^{235} \mathrm{U}=53.8 \%$, ${ }^{239} \mathrm{Pu}=32.8 \%, \mathrm{Pu}^{241}=5.6 \%$ and $\mathrm{U}^{238}=7.8 \%$, in fractions of fissions per isotope. We note here that the published Bugey- 4 cross section calculation does not account for the contribution of long-lived fission products (offequilibrium effects). The reference electron spectra used for the determination of $S_{\text {tot }}$ were acquired after an irradiation time in the ILL reactor flux of less than 1.5 day for all isotopes. But in the Bugey experiments at a commercial PWR the irradiation time scale was typically about one year. $S_{\text {tot }}$ should thus be corrected for the accumulation of long-lived fission products in the low energy part of the spectrum. We had to turn off these effects in our computation to recover the $\sigma_{f}^{\text {pred,old }}$ value. Including these effects in our calculations would lead to a $+1.0 \%$ 


\begin{tabular}{c|c|c}
\hline \hline & old [3] & new \\
\hline$\sigma_{f, 235 \mathrm{U}}^{\text {pred }}$ & $6.39 \pm 1.9 \%$ & $6.61 \pm 2.11 \%$ \\
$\sigma_{f, 239}^{\text {pred }}$ & $4.19 \pm 2.4 \%$ & $4.34 \pm 2.45 \%$ \\
$\sigma_{f, 238 \mathrm{U}}^{\text {pred }}$ & $9.21 \pm 10 \%$ & $10.10 \pm 8.15 \%$ \\
$\sigma_{f, 241 \mathrm{Pu}}^{\text {pred }}$ & $5.73 \pm 2.1 \%$ & $5.97 \pm 2.15 \%$ \\
\hline$\sigma_{f}^{\text {pred }}$ & $5.824 \pm 2.7 \%$ & $6.102 \pm 2.7 \%$ \\
\hline$\sigma_{f}^{\text {Bugey }}$ & \multicolumn{2}{|c}{$5.752 \pm 1.4 \%[3]$} \\
$\sigma_{f}^{\text {Bugey }} / \sigma_{f}^{\text {pred }}$ & $0.987 \pm 1.4 \% \pm 2.7 \%$ & $0.943 \pm 1.4 \% \pm 2.7 \%$ \\
\hline \hline
\end{tabular}

TABLE I. Individual cross sections per fission per fissile isotope, $\sigma_{f, k}^{\text {pred }}$. The slight differences in the ratios are caused by differences in off-equilibrium effects. Results of the comparison of $\sigma_{f}^{\text {Bugey }}$ at Bugey-4 in units of $10^{-43} \mathrm{~cm}^{2} /$ fission with the former and present prediction, $\sigma_{f}^{\text {pred }}$, are shown.

discrepancy. An over or under-estimation of the irradiation time by 100 days would lead to a systematic error on the off-equilibrium correction below $0.1 \%$.

Computing the ratio of observed versus expected events $R_{\text {Bugey }}=\sigma_{f}^{\text {Bugey }} / \sigma_{f}^{\text {pred }}$, Bugey- 4 concluded that there was good agreement with the V-A theory of weak interactions, and that the neutrino flux emitted by PWR reactors is sufficiently understood to be computed using the parameters of [22, 40, 41].

Applying the new reactor antineutrino spectra we obtain a new value of the cross section per fission of $\sigma_{f}^{\text {pred,new }}=6.102 \pm 0.163$ in units of $10^{-43} \mathrm{~cm}^{2} /$ fission. We thus revised the ratio $R_{\text {Bugey }-4}=0.943 \pm 0.013$ (stat + syst $) \pm 0.025\left(S_{\text {tot }}\right)$, which is now 2.0 standard deviations from the expectation without neutrino oscillations. This creates a tension between the measurement at Bugey-4 and the new predicted cross section per fission. In the next sections we will see that the other reactor neutrino experimental results confirm this anomaly.

\section{B. The ILL neutrino experiment}

In the early eighties the ILL experiment measured the antineutrino induced positron spectrum at a distance of $8.76 \mathrm{~m}$ from the core of the Laue-Langevin fission reactor. Its fuel is highly enriched uranium $\left(93 \%{ }^{235} \mathrm{U}\right)$, simplifying the computation of the predicted antineutrino spectrum rate and shape. In Ref. [2] the ILL experiment reported a ratio of measured to predicted event rates of $R_{\mathrm{ILL}, 80}=0.955 \pm 0.035$ (stat) \pm 0.11 (syst $\left.+S_{\text {tot }}\right)$.

In 1995 this result was revised by part of the ILL collaboration [36]. The 1980 reactor electron spectrum for ${ }^{235} \mathrm{U}$ of Ref. [21] was re-measured in 1984 [22] by the same authors as [21]. The neutron mean lifetime was corrected from $926 \mathrm{~s}$ to $889 \mathrm{~s}$, increasing the predicted cross section by $4 \%$. Moreover in 1990 it was announced that the operating power of the ILL reactor had been incorrectly reported at the time of the neutrino experiment, by $+9.5 \%$. This reanalysis led to $R_{\mathrm{ILL}, 95}=0.832 \pm 0.029$ (stat) \pm 0.0738 (syst $\left.+S_{\text {tot }}\right), \quad$ excluding the no-oscillation case at $2 \sigma$.

According to the new spectra of Ref. [20] the antineutrino rate is increased by $\sim 3.5 \%$ (see Table II). A slight neutron mean lifetime correction leads to an additional $+0.37 \%$ shift. The new ratio is thus $R_{\mathrm{ILL}, \mathrm{new}}=0.802 \pm 0.028$ (stat) \pm 0.071 (syst $\left.+S_{\text {tot }}\right)$, significantly deviating from its expected value.

\section{Bugey-3, Goesgen, Krasnoyarsk, Rovno, SRP}

We now study the impact of the new antineutrino spectra on experiments at Bugey (called Bugey-3), Goesgen, Rovno, and Krasnoyarsk [4 9], which measured the reactor antineutrino rate at short distances, between $15 \mathrm{~m}$ and $95 \mathrm{~m}$, but less accurately than Bugey-4 [3].

Accounting for new reactor antineutrino spectra 20] the Bugey-3 [4] ratios of observed versus expected events between 1 and $6 \mathrm{MeV}$ decrease by $3.7 \%$. However, we have to apply a further correction to account for offequilibrium effects. Assuming 300 days of irradiation this leads to an additional increase by $+1.0 \%$. The Bugey- 3 ratios become: $0.946 \pm 0.004$ (stat) \pm 0.048 (syst $\left.+S_{\text {tot }}\right)$ at $15 \mathrm{~m}, 0.952 \pm 0.01$ (stat) \pm 0.048 (syst $\left.+S_{\text {tot }}\right)$ at $40 \mathrm{~m}$ and $0.876 \pm 0.126$ (stat) \pm 0.048 (syst $\left.+S_{\text {tot }}\right)$ at $95 \mathrm{~m}$. Note that uncertainties on $S_{\text {tot }}$ are included in the errors quoted by the Bugey- 3 collaboration.

A similar analysis is performed with the Goesgen [5] results. The new Goesgen ratios shift to $0.966 \pm 0.017$ (stat) \pm 0.060 (syst $\left.+S_{\text {tot }}\right)$ at $38 \mathrm{~m}$, $0.991 \pm 0.019$ (stat) \pm 0.062 (syst $\left.+S_{\text {tot }}\right)$ at $45 \mathrm{~m}$ and $0.924 \pm 0.033$ (stat) \pm 0.062 (syst $+S_{\text {tot }}$ ) at $65 \mathrm{~m}$.

In the eighties a series of experiments were performed at the Rovno nuclear power station in the Soviet Union.

In 1988 the collaboration published measurements at $18 \mathrm{~m}$ and $25 \mathrm{~m}$ from the reactor core [6]. Five measurements were performed with two different detectors: an integral detector to measure the absolute cross section per fission of inverse $\beta$-decay (labeled 1I and 2I), and a scintillator spectrometer to measure both the absolute and differential cross section per fission (labeled 1S, 2S, and 3S). The neutron lifetime was taken to be $898.8 \mathrm{~s}$. We accounted for the average fuel composition for each run which was published in [6]. As an indication, the average fuel composition over the five measurements is ${ }^{235} \mathrm{U}=59.6 \%$, ${ }^{239} \mathrm{Pu}=28.3 \%,{ }^{241} \mathrm{Pu}=4.6 \%$ and $\mathrm{U}^{238}=7.5 \%$. The five results of the observed over predicted event ratios are reported in table III At $18 \mathrm{~m}$ the average ratio is shifted from $R_{\text {Rovno } 88,18 \mathrm{~m}, \text { new }}=0.995 \pm 0.060$ (stat + syst $\left.+S_{\text {tot }}\right)$ to $R_{\text {Rovno } 88,18 \mathrm{~m}, \mathrm{new}}=0.944 \pm 0.057$ (stat + syst $\left.+S_{\text {tot }}\right)$.

In 1991 the Rovno integral experiment [7] published a cross section per fission of $\sigma_{f}^{\text {Rovno91 }}=5.85 \pm 0.17$ in units of $10^{-43} \mathrm{~cm}^{2} /$ fission, $18 \mathrm{~m}$ away from a nuclear core with an average fuel composition of ${ }^{235} \mathrm{U}=61.4 \%$, ${ }^{239} \mathrm{Pu}=27.4 \%,{ }^{241} \mathrm{Pu}=3.8 \%$ and $\mathrm{U}^{238}=7.4 \%$. They 
predicted the cross section $\sigma_{f, \text { Rovno91 }}^{\text {pred,old }}=5.94 \pm 0.16$ in units of $10^{-43} \mathrm{~cm}^{2} /$ fission, and thus obtained the ratio $R_{\text {Rovno91,old }}=0.985 \pm 0.037$ (stat + syst $+S_{\text {tot }}$ ). We recomputed the cross section per fission according to the new antineutrino spectra and found $\sigma_{f, \text { Rovno91 }}^{\text {pred,new }}=6.223 \pm 0.17$. The new ratio is thus revised to $R_{\text {Rovno91,new }}=0.940 \pm 0.036$ (stat + syst $\left.+S_{\text {tot }}\right)$. We note that the correction of the neutron mean lifetime contributes $1.8 \%$ to the shift of the ratio.

In 1984 a neutrino experiment operated at the Krasnoyarsk reactors [8], which have an almost pure ${ }^{235} \mathrm{U}$ fuel composition leading to an antineutrino spectrum within $1 \%$ of pure ${ }^{235} \mathrm{U}$, and operate over 50 day cycles. They measured the cross section per fission at two distances, $\sigma_{f}^{\text {Krasno, } 33 \mathrm{~m}}=6.19 \pm 0.36$ at $33 \mathrm{~m}$ and $\sigma_{f}^{\mathrm{Krasno}, 92 \mathrm{~m}}=6.30 \pm 1.28$ at $92 \mathrm{~m}$, in units of $10^{-43} \mathrm{~cm}^{2} /$ fission. They compared it to the predicted cross section of $6.11 \pm 0.2110^{-43} \mathrm{~cm}^{2} /$ fission, based on the Ref. [21] ${ }^{235} \mathrm{U}$ measurement instead of Ref. [22]. Correcting the neutron mean lifetime and using the new antineutrino spectra we obtain a predicted cross section of $6.6110^{-43} \mathrm{~cm}^{2} /$ fission, assuming a pure ${ }^{235} \mathrm{U}$ spectrum. This leads to the ratios $R_{\text {Krasno,33m }}=0.936 \pm 0.054$ ( stat + syst $\left.+S_{\text {tot }}\right)$ and $R_{\text {Krasno, } 92 \mathrm{~m}}=0.953 \pm 0.195$ (stat + syst $\left.+S_{\text {tot }}\right)$, at $33 \mathrm{~m}$ and $92 \mathrm{~m}$, respectively. In 1994 two other measurements were performed $57 \mathrm{~m}$ from the Krasnoyarsk reactors [9]. They measured $\sigma_{f}^{\mathrm{Krasno}, 57 \mathrm{~m}}=6.26 \pm 0.26$ at $57 \mathrm{~m}$, and compared it to their predicted cross section of $6.33 \pm 0.1910^{-43} \mathrm{~cm}^{2} /$ fission, based on Ref. [22] and in agreement with our reevaluation using previous reference antineutrino spectra. Using the new values of Ref. [20] we revise the ratio $R_{\text {Krasno }, 57 \mathrm{~m}}=0.947 \pm 0.047$ (stat + syst $\left.+S_{\text {tot }}\right)$.

From the neutrino pioneering experiments led by F. Reines and C. Cowan [10] to the nineties, a series of reactor antineutrino measurements were performed at the Savannah River Plant (SRP), a U.S production facility for tritium and plutonium. For neutrino energies between 2 and $8 \mathrm{MeV}$ the spectrum difference between SRP and a similar core with pure ${ }^{235} \mathrm{U}$ fuel was estimated to be less than $0.5 \%$. We make use of the latest results published in Ref. [1]. Measurements were reported at two different baselines, $18 \mathrm{~m}$ and $24 \mathrm{~m}$. The new SRP ratios are reevaluated to $0.953 \pm 0.006$ (stat) \pm 0.0353 (syst $\left.+S_{\text {tot }}\right)$ and $1.019 \pm 0.010$ (stat) \pm 0.0377 (syst $+S_{\text {tot }}$ ), respectively.

\section{CHOOZ and Palo Verde}

Based on the good agreement between $\sigma_{f}^{\text {pred,old }}$ and $\sigma_{f}^{\text {Bugey }}$ obtained at Bugey-4 [3], the CHOOZ experiment [13] decided to use the total cross section per fission measured at Bugey-4, correcting a posteriori for the dif- ferent averaged fuel composition according to:

$$
\sigma_{f}^{\text {Chooz }}=\sigma_{f}^{\text {Bugey }}+\sum_{k}\left(f_{k}^{\text {Chooz }}-f_{k}^{\text {Bugey }}\right) \sigma_{f, k}^{\text {pred,old }},
$$

where $f_{k}^{\text {Chooz }}$ and $f_{k}^{\text {Bugey }}$ are the contributions of the $k^{\text {th }}$ isotope to the total amount of fissions at the $\mathrm{CHOOZ}$ and Bugey-4 experiments [37]. This explicitly means that the expected number of events was computed using $\sigma_{f}^{\text {Bugey }}$ rather than $\sigma_{f}^{\text {pred,old }}$, thus absorbing a $-1.3 \%$ difference on the overall normalization (see Table \). This also has the effect of reducing the error on the neutrino detection rate from $2.7 \%$ to $1.6 \%$ (including the uncertainty on the fission contributions $f_{k}$ ). Accounting for an uncertainty on off-equilibrium effects, CHOOZ quoted a final neutrino spectrum error of $1.9 \%$. As shown above, the new values from Ref. 20] lead to an increase of $\sigma_{f}^{\text {pred,new }}=1.048 \sigma_{f}^{\text {pred,old }}$. With this sizeable discrepancy between measured and computed cross sections, the CHOOZ experiment cannot rely anymore on the effective cross section per fission measured at Bugey-4, assuming no-oscillation at baselines of less than a few tens of meters. We thus revise the ratio to $R_{\text {Chooz }}=0.961 \pm 0.027$ (stat) \pm 0.032 (syst $+S_{\text {tot }}$ ) (see Table II), where the $3.3 \%$ systematic error now includes the $2.7 \%$ uncertainty on $\sigma_{f}^{\text {pred,new }}$ (See Appendix A).

A crude analysis of the impact of Ref. [20] on Palo Verde data 44 leads to the modification of the average ratio of detected versus expected $\bar{\nu}_{e}$ by roughly $-3.5 \%$, leading to $R_{\text {PaloVerde }}=0.975 \pm 0.023$ (stat) \pm 0.055 (syst $+S_{\text {tot }}$ ).

We also note that Palo Verde's uncertainty would increase from $5.3 \%$ in Ref. [44] to $5.6 \%$ according to our prescription.

\section{THE REACTOR ANTINEUTRINO ANOMALY}

\section{A. Rate information}

Up to now we independently studied the results of the main reactor neutrino experiments using a new value of the cross section per fission, $\sigma_{f}^{\text {pred,new }}$. The ratios of observed event rates to predicted event rates, $R=N_{\text {obs }} / N_{\text {pred }}$, are summarized in Table [I] We observe a general systematic shift more or less significantly below unity. These reevaluations unveil a new reactor antineutrino anomaly, clearly illustrated in Figure 5, but still to be explained. In order to quantify the statistical significance of the anomaly we can compute the weighted average of the ratios of expected over predicted rates, for all short baseline reactor neutrino experiments (including their possible correlations).

We consider the following experimental rate information: Bugey-4 and Rovno91, the three Bugey-3 experiments, the three Goesgen experiments and the ILL experiment, the three Krasnoyarsk experiments, the two 


\begin{tabular}{c|c|c|c|c|c|c|c|c|c|c|c|c}
\hline \hline$\#$ & result & Det. type & $\tau_{n}(\mathrm{~s})$ & ${ }^{235} \mathrm{U}$ & ${ }^{239} \mathrm{Pu}$ & ${ }^{238} \mathrm{U}$ & ${ }^{241} \mathrm{Pu}$ & old & new & err(\%) & corr $(\%)$ & $\mathrm{L}(\mathrm{m})$ \\
\hline 1 & Bugey-4 & ${ }^{3} \mathrm{He}+\mathrm{H}_{2} \mathrm{O}$ & 888.7 & 0.538 & 0.328 & 0.078 & 0.056 & 0.987 & 0.942 & 3.0 & 3.0 & 15 \\
2 & ROVNO91 & ${ }^{3} \mathrm{He}+\mathrm{H}_{2} \mathrm{O}$ & 888.6 & 0.614 & 0.274 & 0.074 & 0.038 & 0.985 & 0.940 & 3.9 & 3.0 & 18 \\
\hline 3 & Bugey-3-I & ${ }^{6} \mathrm{Li}-\mathrm{LS}$ & 889 & 0.538 & 0.328 & 0.078 & 0.056 & 0.988 & 0.946 & 4.8 & 4.8 & 15 \\
4 & Bugey-3-II & ${ }^{6} \mathrm{Li}-\mathrm{LS}$ & 889 & 0.538 & 0.328 & 0.078 & 0.056 & 0.994 & 0.952 & 4.9 & 4.8 & 40 \\
5 & Bugey-3-III & ${ }^{6} \mathrm{Li}-\mathrm{LS}$ & 889 & 0.538 & 0.328 & 0.078 & 0.056 & 0.915 & 0.876 & 14.1 & 4.8 & 95 \\
\hline 6 & Goesgen-I & ${ }^{3} \mathrm{He}+\mathrm{LS}$ & 897 & 0.620 & 0.274 & 0.074 & 0.042 & 1.018 & 0.966 & 6.5 & 6.0 & 38 \\
7 & Goesgen-II & ${ }^{3} \mathrm{He}+\mathrm{LS}$ & 897 & 0.584 & 0.298 & 0.068 & 0.050 & 1.045 & 0.992 & 6.5 & 6.0 & 45 \\
8 & Goesgen-II & ${ }^{3} \mathrm{He}+\mathrm{LS}$ & 897 & 0.543 & 0.329 & 0.070 & 0.058 & 0.975 & 0.925 & 7.6 & 6.0 & 65 \\
9 & $\mathrm{ILL}$ & ${ }^{3} \mathrm{He}+\mathrm{LS}$ & 889 & $\simeq 1$ & - & - & - & 0.832 & 0.802 & 9.5 & 6.0 & 9 \\
\hline 10 & Krasn. I & ${ }^{3} \mathrm{He}+\mathrm{PE}$ & 899 & $\simeq 1$ & - & - & - & 1.013 & 0.936 & 5.8 & 4.9 & 33 \\
11 & Krasn. II & ${ }^{3} \mathrm{He}+\mathrm{PE}$ & 899 & $\simeq 1$ & - & - & - & 1.031 & 0.953 & 20.3 & 4.9 & 92 \\
12 & Krasn. III & ${ }^{3} \mathrm{He}+\mathrm{PE}$ & 899 & $\simeq 1$ & - & - & - & 0.989 & 0.947 & 4.9 & 4.9 & 57 \\
\hline 13 & SRP I & $\mathrm{Gd}-\mathrm{LS}$ & 887 & $\simeq 1$ & - & - & - & 0.987 & 0.952 & 3.7 & 3.7 & 18 \\
14 & SRP II & $\mathrm{Gd}-\mathrm{LS}$ & 887 & $\simeq 1$ & - & - & - & 1.055 & 1.018 & 3.8 & 3.7 & 24 \\
\hline 15 & ROVNO88-1I & ${ }^{3} \mathrm{He}+\mathrm{PE}$ & 898.8 & 0.607 & 0.277 & 0.074 & 0.042 & 0.969 & 0.917 & 6.9 & 6.9 & 18 \\
16 & ROVNO88-2I & ${ }^{3} \mathrm{He}+\mathrm{PE}$ & 898.8 & 0.603 & 0.276 & 0.076 & 0.045 & 1.001 & 0.948 & 6.9 & 6.9 & 18 \\
17 & ROVNO88-1S & $\mathrm{Gd}-\mathrm{LS}$ & 898.8 & 0.606 & 0.277 & 0.074 & 0.043 & 1.026 & 0.972 & 7.8 & 7.2 & 18 \\
18 & ROVNO88-2S & $\mathrm{Gd}-\mathrm{LS}$ & 898.8 & 0.557 & 0.313 & 0.076 & 0.054 & 1.013 & 0.959 & 7.8 & 7.2 & 25 \\
19 & ROVNO88-3S & Gd-LS & 898.8 & 0.606 & 0.274 & 0.074 & 0.046 & 0.990 & 0.938 & 7.2 & 7.2 & 18 \\
\hline \hline
\end{tabular}

TABLE II. $N_{\text {obs }} / N_{\text {pred }}$ ratios based on old and new spectra. Off-equilibrium corrections have been applied when justified. The err column is the total error published by the collaborations including the error on $S_{\text {tot }}$, the corr column is the part of the error correlated among multiple-baseline experiments, or experiments using the same detector. This table is used to construct the covariance matrix used in Eq. 10.

Savannah River results (SRP), and the five Rovno88 experiments. $\overrightarrow{\mathrm{R}}$ is the corresponding vector of 19 ratios of observed to predicted event rates. We assume a $2.0 \%$ systematic uncertainty fully correlated among all 19 ratios. This choice is motivated by the common normalization uncertainty of the corresponding beta-spectra measured in 21 24]. We considered the ratios and relative errors gathered in Table II In order to account for the potential experimental correlations, we fully correlated the experimental errors of Bugey- 4 and Rovno91, of the three Goesgen and the ILL experiments, the three Krasnoyarsk experiments, the five Rovno 88 experiments, and the two SRP results. We also fully correlated the Rovno 88 (1I and 2I) results with Rovno91, and we added an arbitrary 50\% correlation between the Rovno88 (1I and 2I) and the Bugey-4 measurement. We motivated these latest correlations by the use of similar or identical integral detectors. We stress here that in this publication we only used the error budget published by the collaborations, without any change. We then obtain the covariance matrix $\mathrm{W}$ of the ratios. In Figure 1 we show the corresponding correlation matrix with labels detailed in Table II

All the above-mentioned experiments published ratios of measured to expected event rates. While the rates themselves can be considered to follow Gaussian distributions, the ratio of two Gaussian variables does not. This may lead to overestimating the statistical significance of the deviation of the ratios from unity, as was pointed out by James in response to a paper by Reines et al. [66]. In order to check our results, we performed

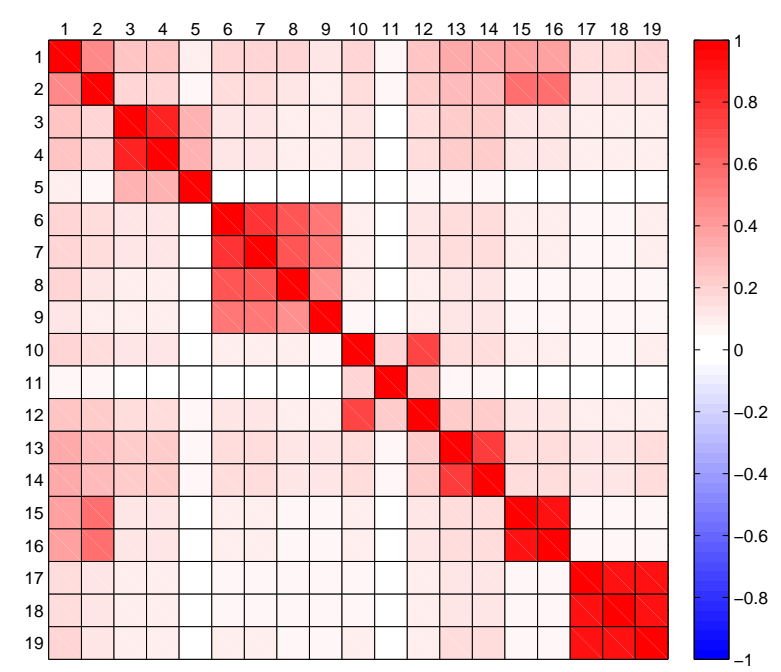

FIG. 1. Correlation matrix of 19 measurements of reactor neutrino experiments operating at short baselines. Experiment labels are given in Table II

simple Monte-Carlo simulations: each experiment's observed and expected rates were simulated within their quoted errors, including the correlations between exper- 
iments shown in the covariance matrix W.

We then calculated the best-fit average ratio $r_{\text {best }}$ for each simple Monte-Carlo experiment by minimizing the $\chi^{2}$ function with respect to $r$ :

$$
(r-\overrightarrow{\mathrm{R}})^{T} W^{-1}(r-\overrightarrow{\mathrm{R}})
$$

and obtained the distribution of $r_{\text {best }}$ (we note $\mu$ its mean in the following paragraph). We found that it is almost Gaussian, but with slightly longer tails, which we decided to take into account in our calculations (in contours that appear later in this article we enlarged the error bars). If we only consider experiments with baselines $<100 \mathrm{~m}$ to get rid of a possible $\left(\theta_{13}, \Delta m_{31}^{2}\right)$ driven oscillation effect at Palo Verde or $\mathrm{CHOOZ}$, with the old antineutrino spectra the mean ratio is $\mu=0.976 \pm 0.024$, and the fraction of simple MonteCarlo experiments with $r \geq 1$ is $17.1 \%(-0.95 \sigma$ from expectation). With the new antineutrino spectra, we obtain $\mu=0.943 \pm 0.023$, and the fraction of simple MonteCarlo experiments with $r \geq 1$ is $1.3 \%$, corresponding to a $-2.2 \sigma$ effect (while a simple calculation assuming normality would lead to $-2.4 \sigma$ ). Clearly the new spectra induce a statistically significant deviation from the expectation. In the following we define an experimental cross section $\sigma_{f}^{\text {ano }}=0.943 \times \sigma_{f}^{\text {pred,new }} 10^{-43} \mathrm{~cm}^{2} /$ fission. With the new antineutrino spectra, we observe that for the data sample the minimum $\chi^{2}$ is $\chi_{\text {min,data }}^{2}=19.6$. The fraction of simple Monte-Carlo experiments with $\chi_{\text {min }}^{2}<\chi_{\text {min,data }}^{2}$ is $25 \%$, showing that the distribution of experimental ratios in $\overrightarrow{\mathrm{R}}$ around the mean value is representative given the correlations.

We will now discuss the possible explanations of this deviation from unity: an erroneous prediction of the antineutrino flux from the reactors, or a correlated artifact in the experiments, or a real physical effect if both previous cases are excluded.

Due to the importance of the antineutrino rate increase we suggest that independent nuclear physics groups should perform similar computations. We also consider that new measurements of the electron spectra of irradiated fissile isotopes would help clarifying the anomaly. All cross sections of reactions used for the absolute and relative normalizations of the ILL electron spectra have been checked and found in agreement with the published values within error bars. A more complete discussion on the evaluation of the normalization of reactor antineutrino spectra based on the $a b$ initio method will be published later in 51].

Assuming the correctness of $\sigma_{f}^{\text {pred,new }}$ the anomaly could be explained by a common bias in all reactor neutrino experiments. The measurements used one of two techniques, scintillator counters and integral detectors. The Bugey-3 experiment [4] used 3 identical detection modules, each of 600 liters, filled with ${ }^{6} \mathrm{Li}$-loaded liquid scintillator. Bugey-3 recorded 120,000 neutrino interactions. The Bugey-4 experiment [3] used the Rovno91 7] integral type detector, but increasing the antineutrino rate by a factor of three. A similar detector was used for two integral measurements Rovno88 $1 \mathrm{I}$ and 2I [7]. In such detectors, based on ${ }^{3} \mathrm{He}$-filled counters, the total number of antineutrino interactions is measured by detecting only the neutrons from reaction Eq. 3. The Goesgen experiment [5] used a detector nearly identical to the one used for the ILL neutrino experiment [2], but with the additional feature of position sensitivity. More than 10,000 neutrino events were recorded at the three detector locations. The detector contained liquid scintillator surrounded by ${ }^{3} \mathrm{He}$-filled wire chambers for neutron detection. Both the positron and the neutron from reaction Eq. 3 were detected. Krasnoyarsk [8, 9] used an integral detector with a scintillation section. The Savannah River experiments considered in this article used a scintillator counter [11].

Neutrons were tagged either by their capture in metalloaded scintillator, or in proportional counters, thus leading to two distinct systematics. As far as the neutron detection efficiency calibration is concerned, we note that different types of radioactive sources emitting $\mathrm{MeV}$ or sub-MeV neutrons were used (Am-Be, ${ }^{252} \mathrm{Cf}, \mathrm{Sb}-\mathrm{Pu}, \mathrm{Pu}-$ $\mathrm{Be})$.

It should be mentioned that the Krasnoyarsk, ILL, and SRP experiments operated with nuclear fuel such that the difference between the real antineutrino spectrum and that of pure ${ }^{235} \mathrm{U}$ was less than $1.5 \%$. They reported similar deficits to those observed at other reactors operating with a mixed fuel of ${ }^{235} \mathrm{U},{ }^{239} \mathrm{Pu},{ }^{241} \mathrm{Pu}$ and ${ }^{238} \mathrm{U}$. Hence the anomaly cannot be associated with a single fissile isotope.

All the elements discussed above argue against a trivial bias in the experiments, but a detailed analysis of the most sensitive of them, involving experts, would certainly improve the quantification of the anomaly.

As discussed in Section II, in a near future the reactor rate anomaly significance might evolve due to the reevaluation of the cross section prefactor $\kappa$ to $0.396110^{-43} \mathrm{~cm}^{2}$. The averaged ratio would shift to $0.938 \pm 0.023$, leading to a deviation from unity at $99.2 \%$ C.L.

The third kind of possible explanation of the anomaly based on a real physical effect will be detailed in Section $\nabla$

\section{B. Shape information}

In this Section we re-analyze the Bugey-3 and ILL shape information, based on the published data [2, 4]. We will use this information for our combined analysis described in the next section.

\section{Bugey-3}

Based on the analysis of the shape of their energy spectra at different source-detector distances [4, 5], the Goesgen and Bugey-3 measurements exclude oscillations such 
that $0.06<\Delta m^{2}<1 \mathrm{eV}^{2}$ for $\sin ^{2}(2 \theta)>0.05$.

For further analysis we used Bugey-3's $40 \mathrm{~m} / 15 \mathrm{~m}$ ratio data from [4] as it provides the best limit. We followed the steps outlined in [4], building the following $\chi^{2}$ function:

$$
\chi^{2}=\sum_{i=1}^{N=25}\left(\frac{(1+a) R_{\mathrm{th}}^{i}-R_{\mathrm{obs}}^{i}}{\sigma_{i}}\right)^{2}+\left(\frac{a}{\sigma_{a}}\right)^{2},
$$

where $R_{\mathrm{obs}}^{i}$ are our simulated data for Bugey from our tuned simulation and $R_{\mathrm{th}}^{i}$ are our Monte-Carlo expectation in each bin. The $\sigma_{i}$ are the errors reported by the Bugey-3 collaboration, and $a$ is a systematic parameter accounting for the $\sigma_{a}=2 \%$ uncertainty on the relative normalization at $40 \mathrm{~m}$ and $15 \mathrm{~m}$. Figure 16 of [4] shows the $90 \%$ C.L. exclusion contour from a raster scan analysis with this estimator. As can be seen in Figure 2, we adequately reproduce Bugey-3's results.

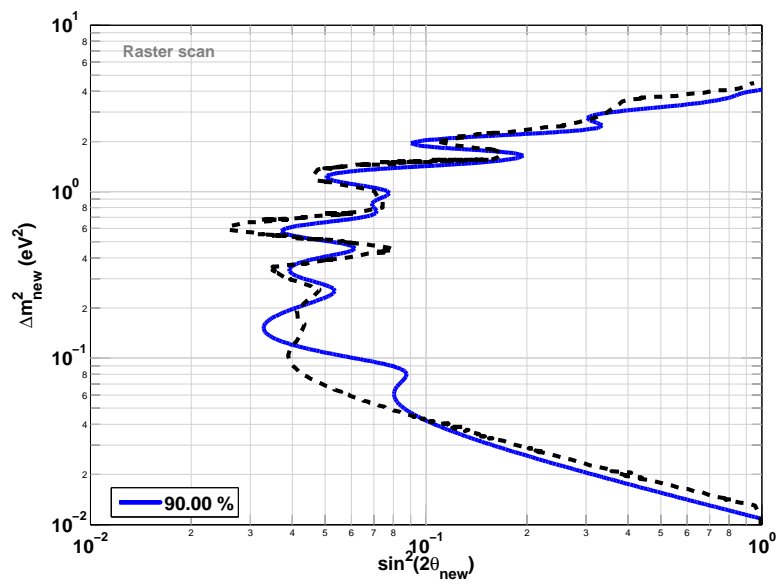

FIG. 2. $90 \%$ C.L. exclusion domains obtained in the $\Delta m^{2}$ $\sin ^{2}(2 \theta)$ plane from a raster scan of Bugey-3's data. Our result (continuous line) is in good agreement with the original result from [4] (dashed line), excluding oscillations such that $0.06<\Delta m^{2}<1 \mathrm{eV}^{2}$ for $\sin ^{2}(2 \theta)>0.05$.

We already note here that in Section $\mathrm{V}$, when combining Bugey-3's shape information with other results, we use the estimator from equation [1] but we perform a standard global scan (i.e. the minimization is performed over the entire $\Delta m^{2}, \sin ^{2}(2 \theta)$ plane). We only perform a raster scan here to show our agreement with the collaboration's original analysis.

\section{2. $I L L$}

As already noted in Ref. [36] the data from ILL showed a spectral deformation compatible with an oscillation pattern in their measured over predicted events ratio. It should be mentioned that the parameters best fitting the data reported by the authors of Ref. [36] were $\Delta m^{2}=2.2 \mathrm{eV}^{2}$ and $\sin ^{2}(2 \theta)=0.3$.
We reanalyzed the data of Ref. [36] in order to include the ILL shape-only information in our analysis of the reactor antineutrino anomaly. We built the following shape-only estimator

$$
\chi_{\mathrm{ILL}, \text { shape }}^{2}=\sum_{i=1}^{N=16}\left(\frac{(1+a) R_{\mathrm{th}}^{i}-R_{\mathrm{obs}}^{i}}{\sigma_{i}}\right)^{2}
$$

where $R_{\mathrm{obs}}^{i}$ are the measured ratios in each energy bin, and $R_{\mathrm{th}}^{i}$ are our Monte-Carlo expectation in each bin. $a$ is a free parameter in the fit, which renders this estimator completely insensitive to any normalization information. It is therefore only sensitive to the shape of the distribution.

$\sigma_{i}$ is the total error in each bin: we added in quadrature the statistical error and a systematic error of $11 \%$. It was difficult to extract the magnitude of this shape-only systematic error from published information. We combined our $\chi_{\text {ILL,shape }}^{2}$ estimator with the rate-only estimator, and verified that with this value of the systematic, we could reproduce the ILL contours of both papers: when using $R_{\mathrm{ILL}, 80}$ we reproduced the contour in Figure 14 of Ref. 2], and when using $R_{\mathrm{ILL}, 95}$ we reproduced that of Ref. 36] which excludes the no-oscillation hypothesis at $2 \sigma$. A systematic error of $11 \%$ is consistent with Figure 13 of Ref. [2]. It is also a conservative treatment of the shape-only information: with such an error the data are compatible with the no-oscillation hypothesis at $1 \sigma$. Figure 3 shows the data points from [36], along with our best fit from the shape-only estimator $\left(\left|\Delta m^{2}\right| \sim 2.3 \mathrm{eV}^{2}\right.$, $\left.\sin ^{2}(2 \theta) \sim 0.24\right)$, and the no-oscillation line for comparison.

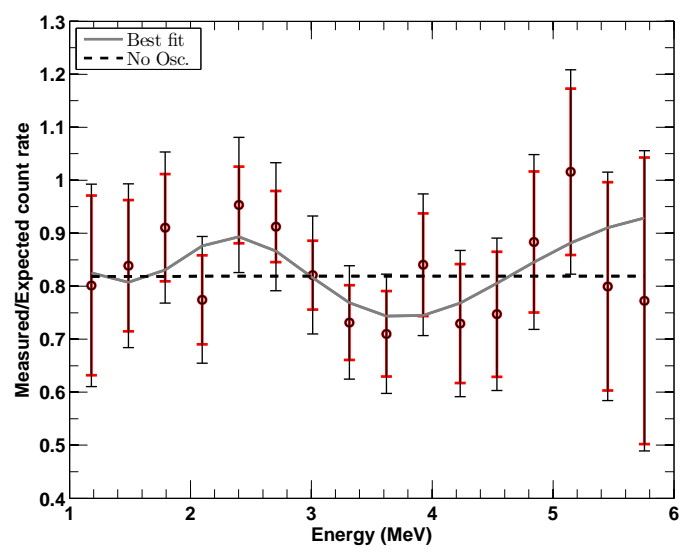

FIG. 3. Ratio of measured to expected positron energy spectra of the ILL neutrino experiment (data points extracted from [36]). We show the best fit line with oscillations, along with the no-oscillation line, from our shape-only fit. The short error bars are statistical and the longer ones include the $11 \%$ systematic error described in the text. 


\section{THE FOURTH NEUTRINO HYPOTHESIS}

In this section we discuss the compatibility of the reactor antineutrino anomaly with the existence of a fourth non-standard neutrino, corresponding in the flavor basis to the existence of a sterile neutrino $\nu_{s}$ (see [1] and references therein). The motivation is the explanation of the antineutrino deficit by an oscillation of electron neutrinos into a new neutrino state with a large $\Delta m_{\text {new }}^{2}$ value.

For simplicity we restrict our analysis to the $3+1$ fourneutrino scheme in which there is a group of three active neutrino masses separated from an isolated neutrino mass, such that $\left|\Delta m_{\text {new }}^{2}\right| \gg 10^{-2} \mathrm{eV}^{2}$. The latter would be responsible for very short baseline reactor neutrino oscillations. For energies above the inverse beta decay threshold and baselines below $2 \mathrm{~km}$, we adopt the approximated oscillation formula from Ref. [52]:

$$
\begin{aligned}
P_{e e}= & 1-\cos ^{4} \theta_{\text {new }} \sin ^{2}\left(2 \theta_{13}\right) \sin ^{2}\left(\frac{\Delta m_{31}^{2} L}{4 E_{\bar{\nu}_{e}}}\right)- \\
& \sin ^{2}\left(2 \theta_{\text {new }}\right) \sin ^{2}\left(\frac{\Delta m_{\text {new }}^{2} L}{4 E_{\bar{\nu}_{e}}}\right) .
\end{aligned}
$$

In our analyses the well known solar driven oscillation effects are negligible. The contribution of the atmospheric driven oscillation is negligible at distances $<100 \mathrm{~m}$. It is worth noting that we are not sensitive to any sterile neutrino mass hierarchy in the mass range considered.

The ILL experiment may have seen a hint of oscillation in their measured positron energy spectrum (see Section IIIB), but Bugey-3's results do not point to any significant spectral distortion more than $15 \mathrm{~m}$ away from the antineutrino source. Hence, in a first approximation, hypothetical oscillations could be seen as an energy-independent suppression of the $\bar{\nu}_{e}$ rate by a factor of $\frac{1}{2} \sin ^{2}\left(2 \theta_{\text {new }, \mathrm{R}}\right)$, thus leading to $\Delta m_{\text {new, } \mathrm{R}}^{2} \gtrsim 1 \mathrm{eV}^{2}$ and accounting for Bugey-3 and Goesgen shape analyses [4, 5]. Considering the weighted averaged of all reactor experiments we get an estimate of the mixing angle, $\sin ^{2}\left(2 \theta_{\text {new }, \mathrm{R}}\right) \sim 0.115$.

Let us now fit the sterile neutrino hypothesis to the data (baselines below $2 \mathrm{~km}$ ) by minimizing the leastsquares function

$$
\left(P_{e e}-\overrightarrow{\mathrm{R}}\right)^{T} W^{-1}\left(P_{e e}-\overrightarrow{\mathrm{R}}\right)
$$

assuming $\sin ^{2}\left(2 \theta_{13}\right)=0$.

Figure 4 provides the results of the fit in the $\sin ^{2}\left(2 \theta_{\text {new }}\right)-\Delta m_{\text {new }}^{2}$ plane, including the reactor experiment rate information as well as the $40 \mathrm{~m} / 15 \mathrm{~m}$ Bugey-3 spectral shape constraint presented in Figure 2 . The latter leads to stringent oscillation constraints for $\left|\Delta m_{\text {new,R }}^{2}\right| \leq 1 \mathrm{eV}^{2}$, since no spectral distortion was observed in the ratio $40 \mathrm{~m} / 15 \mathrm{~m}$. The fit to the data indicates that $\left|\Delta m_{\text {new,R }}^{2}\right|>0.2 \mathrm{eV}^{2}$ (95\%) and $\sin ^{2}\left(2 \theta_{\text {new }, \mathrm{R}}\right) \sim 0.1$. Note that if we include a non-zero value of $\sin ^{2}\left(2 \theta_{13}\right)$, as large as the $90 \%$ C.L. CHOOZ

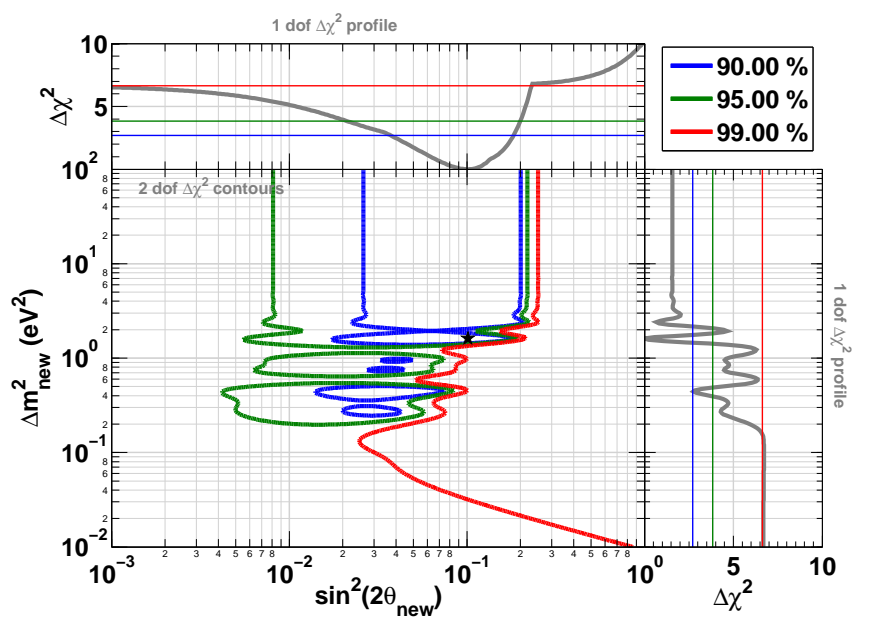

FIG. 4. Allowed regions in the $\sin ^{2}\left(2 \theta_{\text {new }}\right)-\Delta m_{\text {new }}^{2}$ plane obtained from the fit of the reactor neutrino data, without ILL-shape information, but with the stringent oscillation constraint of Bugey- 3 based on the $40 \mathrm{~m} / 15 \mathrm{~m}$ ratios to the $3+1$ neutrino hypothesis, with $\sin ^{2}\left(2 \theta_{13}\right)=0$. The best-fit point is indicated by a star.

bound [13], the contours presented in Figure 4 are only marginally affected.

Ignoring the reactor anomaly leads to an ambiguous interpretation of the $\sim 1-2 \mathrm{~km}$ baseline experiment results constraining $\theta_{13}$. As an example we compare the following two hypotheses: the 3 neutrino mixing scenario with $\sin ^{2}\left(2 \theta_{13}\right)=0.06$ for instance and the $3+1$ neutrino mixing scenario, taking $\sin ^{2}\left(2 \theta_{13}\right)=0, \sin ^{2}\left(2 \theta_{\text {new }, \mathrm{R}}\right)=0.115$ and $\left|\Delta m_{\text {new, }}^{2}\right|>1 \mathrm{eV}^{2}$ (best values of the combined fit shown on Figure [8). Figure [5] displays the data together with the two hypotheses. The red line represents the three active neutrino hypothesis, considering reactor neutrino rates until the $\mathrm{CHOOZ}$ baseline. The p-value of the 3 neutrino mixing hypothesis described above is $17 \%$ (26.9/21 degrees of freedom). The blue line illustrates the $3+1$ neutrino mixing scenario with a large $\left|\Delta m_{\text {new, } \mathrm{R}}^{2}\right|=2.4 \mathrm{eV}^{2}$, leading to a p-value of $55 \%$ (19.4/21 degrees of freedom). We note a tension between the data and the three active neutrino flavor hypothesis, again illustrating the anomaly. Clear preference is given to the $3+1$ neutrino hypothesis since $\Delta \chi^{2} \gtrsim 7$. A similar computation with the former reference spectra would lead to a p-value of $34 \%$ in both cases. This illustrates the impact of our reanalysis with the new antineutrino spectra, updated neutron mean lifetime, and off-equilibrium effects.

At this stage it is tempting to consider the previously noted anomalies affecting other short baseline electron neutrino experiments Gallex, Sage and MiniBooNE, reviewed in Ref. [56]. Our goal is to quantify the compatibility with those anomalies.

We first reanalyzed the Gallex and Sage calibration runs with ${ }^{51} \mathrm{Cr}$ and ${ }^{37} \mathrm{Ar}$ radioactive sources emit- 


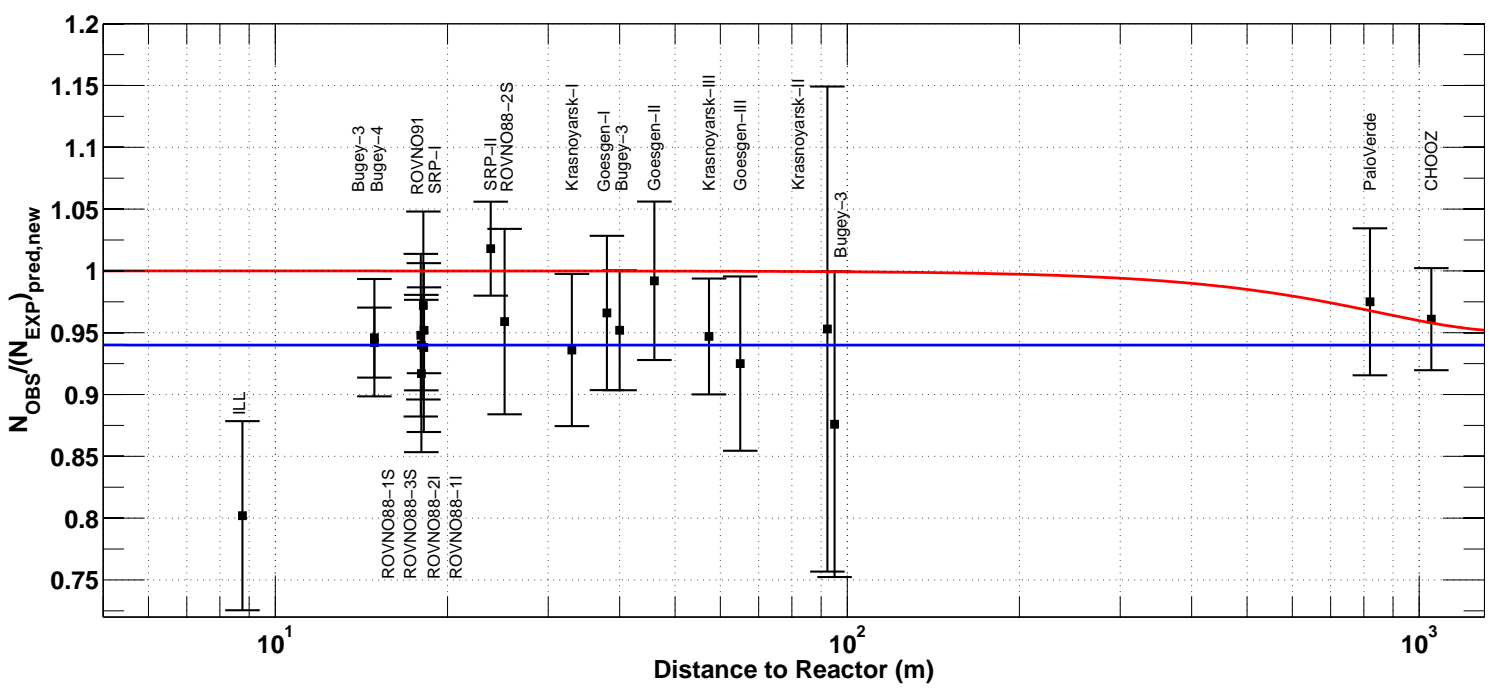

FIG. 5. Illustration of the short baseline reactor antineutrino anomaly. The experimental results are compared to the prediction without oscillation, taking into account the new antineutrino spectra, the corrections of the neutron mean lifetime, and the off-equilibrium effects. Published experimental errors and antineutrino spectra errors are added in quadrature. The mean averaged ratio including possible correlations is $0.943 \pm 0.023$. The red line shows a possible 3 active neutrino mixing solution, with $\sin ^{2}\left(2 \theta_{13}\right)=0.06$. The blue line displays a solution including a new neutrino mass state, such as $\left|\Delta m_{\text {new,R }}^{2}\right| \gg 1 \mathrm{eV}{ }^{2}$ and $\sin ^{2}\left(2 \theta_{\text {new }, \mathrm{R}}\right)=0.12$ (for illustration purpose only).

ting $\sim 1 \mathrm{MeV}$ electron neutrinos. 57], following the methodology developed in Ref. [56, 58]. However we decided to include possible correlations between these four measurements in this present work. Details are given in Appendix B. This has the effect of being slightly more conservative, with the no-oscillation hypothesis disfavored at $97.7 \%$ C.L., instead of $98 \%$ C.L. in Ref. [56]. Gallex and Sage observed an average deficit of $R_{G}=0.86 \pm 0.06(1 \sigma)$. Considering the hypothesis of $\nu_{e}$ disappearance caused by short baseline oscillations we used Eq. (13), neglecting the $\Delta m_{31}^{2}$ driven oscillations because of the very short baselines of order 1 meter. Fitting the data leads to $\left|\Delta m_{\text {new }, \mathrm{G}}^{2}\right|>0.3 \mathrm{eV}^{2}(95 \%)$ and $\sin ^{2}\left(2 \theta_{\text {new }, \mathrm{G}}\right) \sim 0.26$. Combining the reactor antineutrino anomaly with the gallium anomaly gives a good fit to the data and disfavors the no-oscillation hypothesis at $99.7 \%$ C.L. Allowed regions in the $\sin ^{2}\left(2 \theta_{\text {new }}\right)-\Delta m_{\text {new }}^{2}$ plane are displayed in Figure [6 (left). The associated best-fit parameters are $\left|\Delta m_{\text {new }, \mathrm{R} \& \mathrm{G}}^{2}\right|>1.5 \mathrm{eV}^{2}(95 \%)$ and $\sin ^{2}\left(2 \theta_{\text {new,R\&G }}\right) \sim 0.12$.

We then reanalyzed the MiniBooNE electron neutrino excess assuming the very short baseline neutrino oscillation explanation of Ref. [56]. Details of our reproduction of the latter analysis are provided in Appendix B. The best fit values are $\left|\Delta m_{\text {new, MB }}^{2}\right|=1.9 \mathrm{eV}^{2}$ and $\sin ^{2}\left(2 \theta_{\text {new,MB }}\right) \sim 0.2$, but are not significant at 95\% C.L. The no-oscillation hypothesis is only disfavored at the level of $72.4 \%$ C.L., less significant than the reactor and gallium anomalies. Combining the reactor antineutrino anomaly with our MiniBooNE re-

\begin{tabular}{c|c|c|c}
\hline \hline Experiment(s) & $\sin ^{2}\left(2 \theta_{\text {new }}\right)$ & $\Delta m_{\text {new }}^{2}\left(\mathrm{eV}^{2}\right)$ & C.L. $(\%)$ \\
\hline Reactors (no ILL-S,R $\left.\mathrm{R}^{*}\right)$ & $0.02-0.20$ & $>0.40$ & 96.5 \\
Gallium (G) & $>0.06$ & $>0.13$ & 96.1 \\
MiniBooNE (M) & - & - & 72.4 \\
ILL-S & - & - & 68.1 \\
\hline $\mathrm{R}^{*}+\mathrm{G}$ & $0.05-0.22$ & $>1.45$ & 99.7 \\
$\mathrm{R}^{*}+\mathrm{M}$ & $0.04-0.20$ & $>1.45$ & 97.6 \\
$\mathrm{R}^{*}+$ ILL-S & $0.02-0.21$ & $>0.23$ & 95.3 \\
\hline All & $0.06-0.22$ & $>1.5$ & 99.8 \\
\hline \hline
\end{tabular}

TABLE III. Best fit parameter intervals or limits at 95\% C.L. for $\sin ^{2}\left(2 \theta_{\text {new }}\right)$ and $\left|\Delta m_{\text {new }}^{2}\right|$ parameters, and significance of the sterile neutrino oscillation hypothesis in $\%$, for different combinations of the reactor experimental rates only $\left(\mathrm{R}^{*}\right)$, the ILL-energy spectrum information (ILL-S), the gallium experiments (G), and MiniBooNE- $\nu(\mathrm{M})$ re-analysis of Ref. [56]. We quantify the difference between the $\sin ^{2}\left(2 \theta_{\text {new }}\right)$ constraints obtained from the reactor and gallium results. Following prescription of Ref. 77], the parameter goodness-of-fit is $27.0 \%$, indicating reasonable agreement between the neutrino and antineutrino data sets (see Appendix B).

analysis leads to a good fit with the sterile neutrino hypothesis and disfavors the absence of oscillations at $98.5 \%$ C.L., dominated by the reactor experiments data. Allowed regions in the $\sin ^{2}\left(2 \theta_{\text {new }}\right)-\Delta m_{\text {new }}^{2}$ plane are displayed in Figure [6 (right). The associated bestfit parameters are $\left|\Delta m_{\text {new, R\&MB }}^{2}\right|>0.4 \mathrm{eV}^{2}(95 \%)$ and $\sin ^{2}\left(2 \theta_{\text {new }, \mathrm{R} \& \mathrm{MB}}\right) \sim 0.1$. 

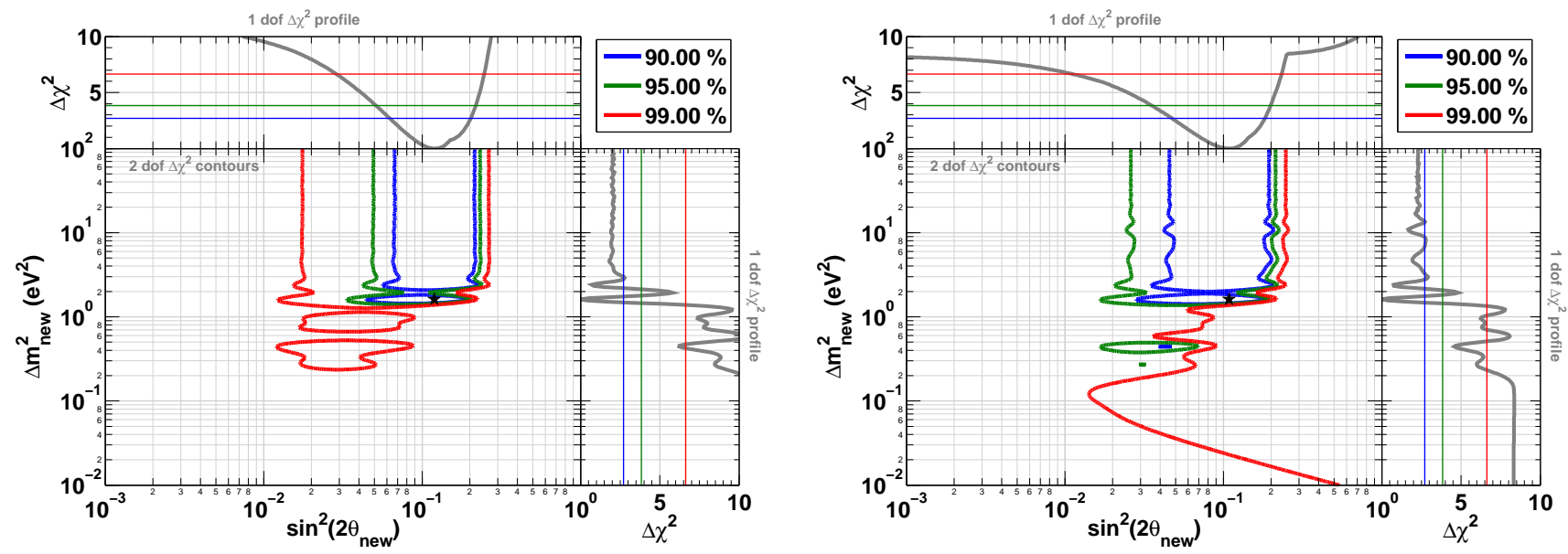

FIG. 6. Allowed regions in the $\sin ^{2}\left(2 \theta_{\text {new }}\right)-\Delta m_{\text {new }}^{2}$ plane obtained from the fit of the reactor neutrino data to the $3+1$ neutrino hypothesis, with $\sin ^{2}\left(2 \theta_{13}\right)=0$. The left panel is the combination of the reactors and the gallium experiment calibration results with ${ }^{51} \mathrm{Cr}$ and ${ }^{37} \mathrm{Ar}$ radioactive sources. The right panel is the combination of the reactors and our reanalysis of the MiniBooNE data following the method of Ref. [56]. In both cases the ILL energy spectrum information is not included.

Our ILL re-analysis, including only the energy spectrum shape, leads to the allowed regions in the $\sin ^{2}\left(2 \theta_{\text {new }}\right)-\Delta m_{\text {new }}^{2}$ plane presented in Figure 7. We notice a hint of neutrino oscillations such that $\left|\Delta m_{\text {new,ILL-shape }}^{2}\right|>1 \mathrm{eV}^{2}$ and $\sin ^{2}\left(2 \theta_{\text {new,ILL-shape }}\right) \sim 0.2$, in agreement with our fourth neutrino hypothesis, but still compatible with the absence of oscillations at the $1 \sigma$ level. Figure 3 is our reproduction of the illustration 3 of Ref. 2]; we superimposed the oscillation pattern that would be induced by neutrino oscillations at our best fit (combined analysis). The ILL positron spectrum is thus in agreement with the oscillation parameters found independently in our re-analyses, mainly based on rate information. Because of the differences in the systematic effects in the rate and shape analyses, this coincidence is in favor of a true physical effect rather than an experimental anomaly. As a cross check we performed a Monte-Carlo simulation of the ILL and Bugey-3 experiments, including the finite spatial extension of the nuclear reactors and the ILL and Bugey- 3 detectors. We found that the small dimensions of the ILL nuclear core lead to small corrections of the oscillation pattern imprinted on the positron spectrum. However the large extension of the Bugey nuclear core is sufficient to wash out most of the oscillation pattern at $15 \mathrm{~m}$. This explains the absence of shape distortion in the Bugey-3 experiment.

Table III summarizes all the results of our fits of reactor, gallium, and MiniBooNE- $\nu$ data to the sterile neutrino oscillation hypothesis. We observe that all the data sets taken separately are very consistent with one another, pointing to very similar oscillation parameters. We thus performed a global fit to all available data.

The no-oscillation hypothesis is disfavored at 99.8\% C.L. The significance is dominated by the

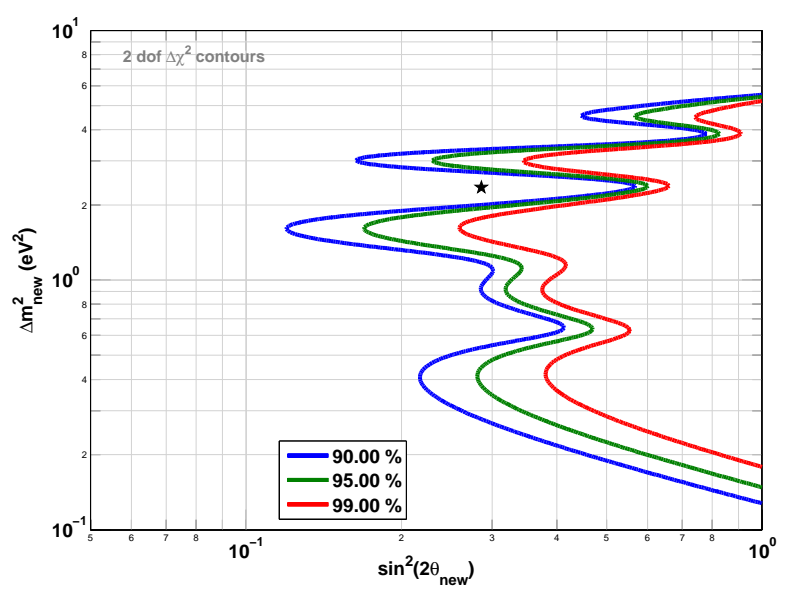

FIG. 7. Allowed regions in the $\sin ^{2}\left(2 \theta_{\text {new }}\right)-\Delta m_{\text {new }}^{2}$ plane obtained from a fit of the ILL energy spectrum shape only. The best fit value reported by the authors of Ref. [36] is very close to our best fit, at $\left|\Delta m_{\text {new }}^{2}\right| \sim 2 \mathrm{eV}^{2}$, but it is worth noting its poor statistical significance, compatible with the absence of oscillations at the $1 \sigma$ level. The best-fit point is indicated by a star.

gallium and reactor data. Allowed regions in the $\sin ^{2}\left(2 \theta_{\text {new }}\right)-\Delta m_{\text {new }}^{2}$ plane are displayed in Figure 8 , together with the marginal $\Delta \chi^{2}$ profiles for $\left|\Delta m_{\text {new }}^{2}\right|$ and $\sin ^{2}\left(2 \theta_{\text {new }}\right)$. The combined fit leads to the following constraints on oscillation parameters: $\left|\Delta m_{\text {new }}^{2}\right|>1.5 \mathrm{eV}^{2}$ $(95 \%$ C.L. $)$ and $\sin ^{2}\left(2 \theta_{\text {new }}\right)=0.14 \pm 0.08$ (95\% C.L. $)$. An embryo of possible consequences of this result will be discussed in Section VIII. 


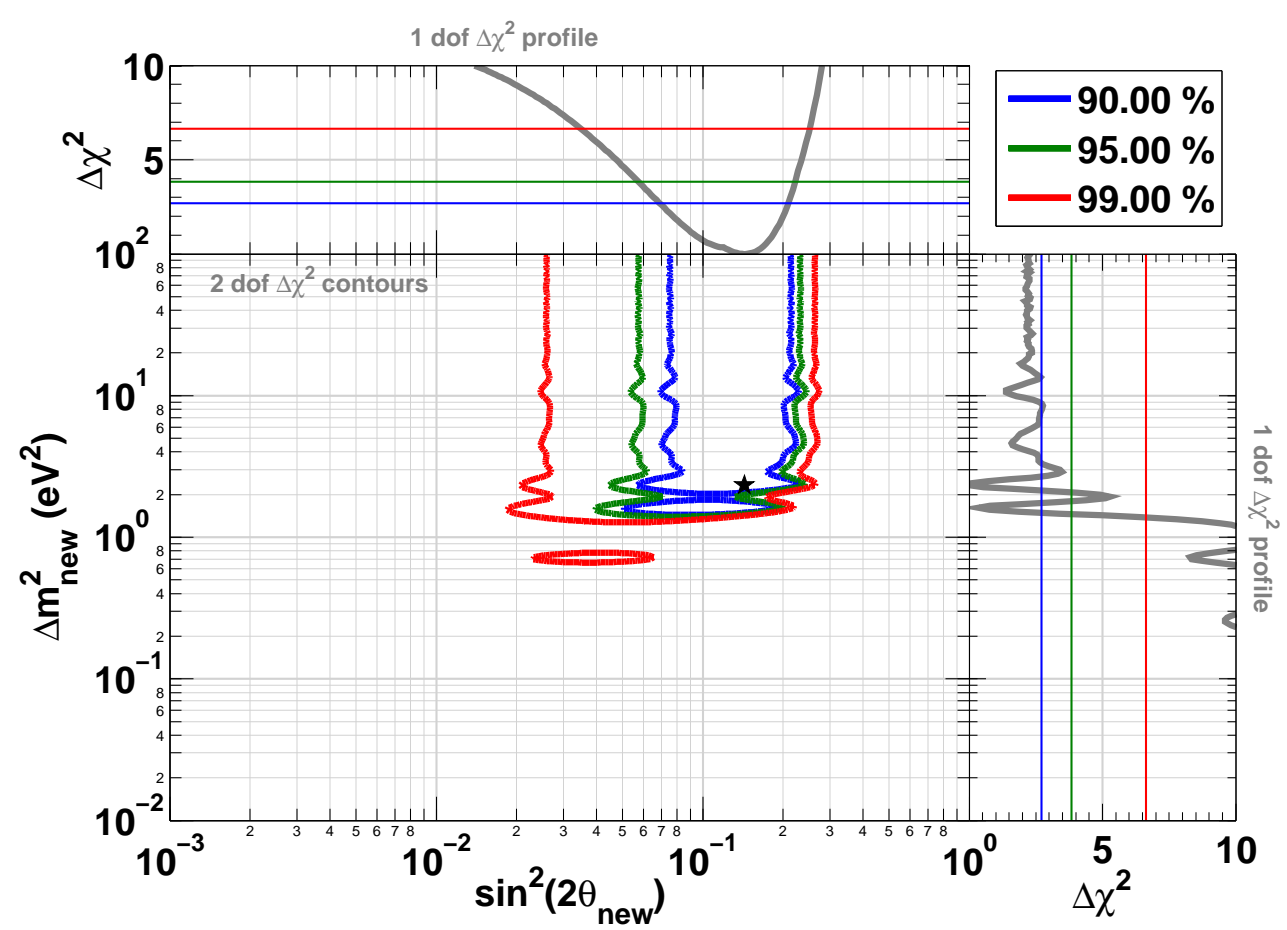

FIG. 8. Allowed regions in the $\sin ^{2}\left(2 \theta_{\text {new }}\right)-\Delta m_{\text {new }}^{2}$ plane from the combination of reactor neutrino experiments, Gallex and Sage calibration sources experiments, MiniBooNE reanalysis of Ref. [56], and the ILL-energy spectrum distortion. The data are well fitted by the $3+1$ neutrino hypothesis, while the no-oscillation hypothesis is disfavored at $99.8 \%$ C.L. The marginal $\Delta \chi^{2}$ profiles for $\left|\Delta m_{\text {new }}^{2}\right|$ and $\sin ^{2}\left(2 \theta_{\text {new }}\right)$ (1 dof) lead to the constraints, $\left|\Delta m_{\text {new }}^{2}\right|>1.5 \mathrm{eV}^{2}(95 \%$ C.L. $)$ and $\sin ^{2}\left(2 \theta_{\text {new }}\right)=0.14 \pm 0.08$ (95\% C.L.).

\section{REVISION OF THE CONSTRAINTS ON $\theta_{13}$}

In this section we discuss the impact of our revision of the results of short baseline $(<100 \mathrm{~m})$ reactor experiments on the constraints on the $\theta_{13}$ mixing angle from CHOOZ [13], KamLAND [17], and their combination. The results depend on the choice of the cross section per fission used to normalize the predicted antineutrino rate, as well as on the neutrino oscillation scheme used in the analysis, i.e. whether to include a non-standard neutrino or not. Assuming the correctness of the neutrino experiments (detected rate), we show that constraints on $\theta_{13}$ could be derived by using $\sigma_{f}^{\text {pred,ano }}$ and the three-active neutrino scenario.

\section{A. $\mathrm{CHOOZ}$}

We first reproduced CHOOZ's background-subtracted results and Monte-Carlo using Ref. [13]. We used the converted neutrino spectra from ILL electron data 22 , 40, 41], using the parameterization from Ref. [47], and the V-A cross section from [29, 39], normalized to the Bugey-4 value as was done in Ref. [13]. Long-lived fission product contributions were also taken into account (at 300 days of irradiation). As in Ref. [13], we accounted for a $\sigma_{\alpha}=2.7 \%$ systematic error on the overall normalization and a $1.1 \%$ error on the energy scale, along with a bin-to-bin uncorrelated uncertainty describing the uncertainty of the neutrino spectrum. Using the original ILL neutrino converted spectra, with the $\sigma_{f}^{\text {Bugey }}$ crosssection we could roughly reproduce the exclusion limit from Ref. 13] (black dashed line on Figure 9) to acceptable precision (gray line on Figure 9) in the relevant $\Delta m_{31}^{2}$ range, although we could not fully imitate every step in the published analysis. In particular we did not use the same statistical treatment, using only a global scan while the CHOOZ collaboration used the FeldmanCousins unified approach to extract the confidence interval.

Let us now revisit the CHOOZ results using the new antineutrino spectra in the three active neutrino framework. We perform the same analysis as before, but computing the expected number of events based on $\sigma_{f}^{\text {pred,new }}$ and its error. $\sigma_{\alpha}$ was thus increased to $3.3 \%$ to account for this effect. This leads to a new exclusion limit, $\sin ^{2}\left(2 \theta_{13}\right)<0.22(1 \mathrm{dof})$ at $90 \%$ C.L. for $\Delta m_{31}^{2}=2.410^{-3} \mathrm{eV}^{2}$. The corresponding exclusion contour is displayed as the blue line on Figure9. In this approach we make use of $\sigma_{f}^{\text {pred,new }}$ and attribute the slight deficit of $\bar{\nu}_{e}$ at CHOOZ to a $\theta_{13}$ driven oscillation, such that $\sin ^{2}\left(2 \theta_{13}\right)=0.11_{-0.08}^{+0.07}$. However this result only 
holds within the three active neutrino mixing framework, under the hypothesis of no-oscillation at very short baselines. But in Section IV we pointed out an anomaly affecting the short baseline reactor neutrino experiment results. In the scenario discussed above with a new neutrino $\left(\Delta m_{\text {new }}^{2}, \theta_{\text {new }}\right)$ we generate oscillations driven by a large $\left|\Delta m_{\text {new }}^{2}\right| \gg \Delta m_{31}^{2}$. In this case CHOOZ measured a combination of the oscillation driven by $\left(\Delta m_{\text {new }}^{2}, \theta_{\text {new }}\right)$ and $\left(\Delta m_{31}^{2}, \theta_{13}\right)$. A comprehensive $3+1$ neutrino analysis would thus be mandatory to constrain $\theta_{13}$ using the new normalization based on $\sigma_{f}^{\text {pred,new }}$, but we will see next that another approach may be used.

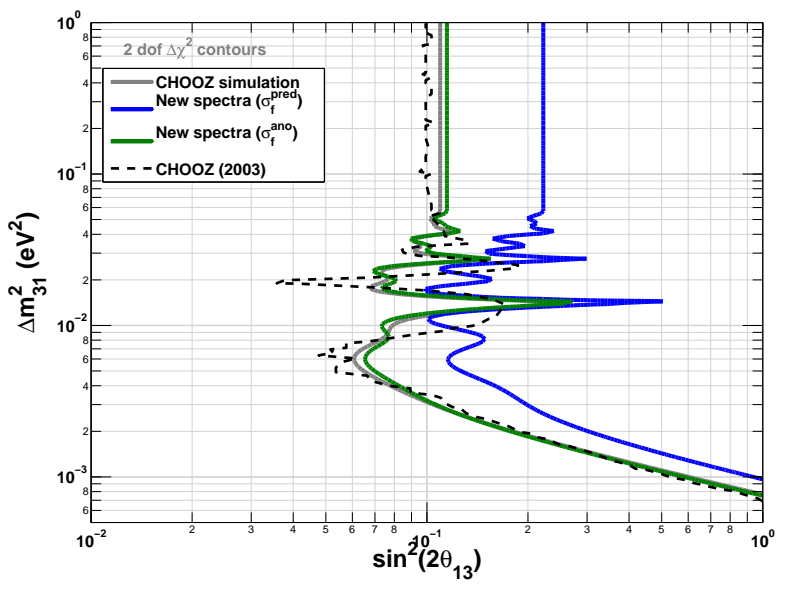

FIG. 9. Our simulation (gray line) roughly reproduces the published CHOOZ exclusion limit [13] represented by the black dashed line. We obtain the blue contour by normalizing the expected no-oscillation antineutrino rate to $\sigma_{f}^{\text {pred,new }}$ and analyzing the data using the three active neutrino oscillations scheme. We then normalize the expected antineutrino event rate to $\sigma_{f}^{\text {ano }}$ to account for possible neutrino oscillations at very short baselines, leading to the green contour. This provides our new constraint on $\sin ^{2}\left(2 \theta_{13}\right)$, accounting for the reactor antineutrino anomaly.

As a matter of fact the CHOOZ experiment used $\sigma_{f}^{\text {Bugey }}$ instead of $\sigma_{f}^{\text {pred,new }}$. The collaboration justified making use of $\sigma_{f}^{\text {Bugey }}$ because of the good agreement with $\sigma_{f}^{\text {pred,old }}$. With our revision of $\sigma_{f}^{\text {pred }}$ this justification does not hold anymore in the 3 neutrino oscillation framework. But it turns out that if we assume non-standard neutrino oscillations in the large $\left|\Delta m_{\text {new }}^{2}\right| \gg 0.1 \mathrm{eV}^{2}$ regime (no spectral distortion), the normalization of $\mathrm{CHOOZ}$ using $\sigma_{f}^{\text {Bugey }} \sim \sigma_{f}^{\text {ano }}$ leads to an estimator of $\sin ^{2}\left(2 \theta_{13}\right)$ which eliminates a possible degeneracy with $\sin ^{2}\left(2 \theta_{\text {new }}\right)$. CHOOZ's strategy was indeed to absorb possible errors in rate predictions, but this methodology holds as well for constraining $\theta_{13}$ on top of an additional, short baseline, averaged oscillation.

Thus a pragmatic approach for constraining $\theta_{13}$ from CHOOZ data is the use of the weighted average of the measured cross sections of all experiments $<100 \mathrm{~m}, \sigma_{f}^{\text {ano }}$.
This leads to a new exclusion limit, $\sin ^{2}\left(2 \theta_{13}\right)<0.10$ (1 dof) at $90 \%$ C.L. for $\Delta m_{31}^{2}=2.410^{-3} \mathrm{eV}^{2}$, slightly lower than CHOOZ's published value [13].

\section{B. KamLAND}

In this section we reevaluate the KamLAND constraint on $\theta_{13}$ in light of the reactor antineutrino anomaly.

Unlike in the original CHOOZ analysis from [13], the KamLAND $\bar{\nu}_{e}$ energy spectrum is calculated using $\sigma_{f}^{\text {pred,old }}$ based on Ref. [22, 40]. But the spectral uncertainty is evaluated from Ref. [3], including off-equilibrium effects. In this case the propagation of the Bugey-4 error on $\sigma_{f}^{\text {pred }}$ only has a marginal impact since it is not among the dominant systematics.

We first reproduced KamLAND's results and MonteCarlo to a very good accuracy exploiting Ref. [17] as well as publicly available information [46]. We used the parameterization of Ref. [47] for $S_{\text {tot }}$, and included off-equilibrium corrections according to Ref. [20]. Special care was taken to include all published backgrounds, known antineutrino sources, especially Korean power reactors, and geoneutrinos. We tuned our simulation to reproduce KamLAND's Monte-Carlo with and without neutrino oscillations (adjusting each effective power of the Japanese power plants). In both cases our simulation agrees with that of KamLAND to better than $1 \%$ in the $1.5-6.5 \mathrm{MeV}$ range.

Confidence levels in the $\left(\tan ^{2} \theta_{12}, \sin ^{2} \theta_{13}\right)$ plane were obtained by minimizing the generic $\chi^{2}$ function:

$$
\chi^{2}=\sum_{i}\left(\frac{Y_{i}-N_{i}(\alpha, \beta)}{Y_{i}}\right)^{2}+\beta^{T} W^{-1} \beta
$$

where the $Y_{i}$ are our simulated data tuned to KamLAND's Monte-Carlo 17]. The free parameters are $\alpha$ $\left(\theta_{12}, \theta_{13}, \Delta m_{21}^{2}\right.$ and geo- $\nu$ rate $)$, and the nuisance parameters, $\beta$, which are the systematics quoted in Ref. [17]. The $N_{i}(\alpha, \beta)$ are our simulation model for all the free parameters. The $W^{-1}$ matrix contains all the systematic uncertainties quoted in Ref. [47].

Figure 10] demonstrates that we could reproduce the best fit and the confidence contours of KamLAND 17] with good accuracy. With the original reactor neutrino spectra $S_{\text {tot, }}$ we obtain a good agreement with KamLAND's published results [17]. Changing the reference spectra $S_{\text {tot }}$ according to Ref. [20], the best fit values and uncertainties on $\tan ^{2} \theta_{12}$ and $\Delta m_{21}^{2}$ are unaffected in a three active neutrino, oscillations framework, but the $\sin ^{2}\left(2 \theta_{13}\right)$ central value is shifted upwards to $\sin ^{2}\left(2 \theta_{13}\right)=0.2_{-0.13}^{+0.14}$, consistent with zero at $1.5 \sigma$. The new $90 \%$ C.L. limit on $\sin ^{2}\left(2 \theta_{13}\right)$ would therefore increase to 0.41 (1 dof), marginalizing over the other fit parameters.

However, as in the case of $\mathrm{CHOOZ}$ discussed above, these results do not take into account the reactor antineutrino anomaly at short baselines. In the $3+1$ neu- 


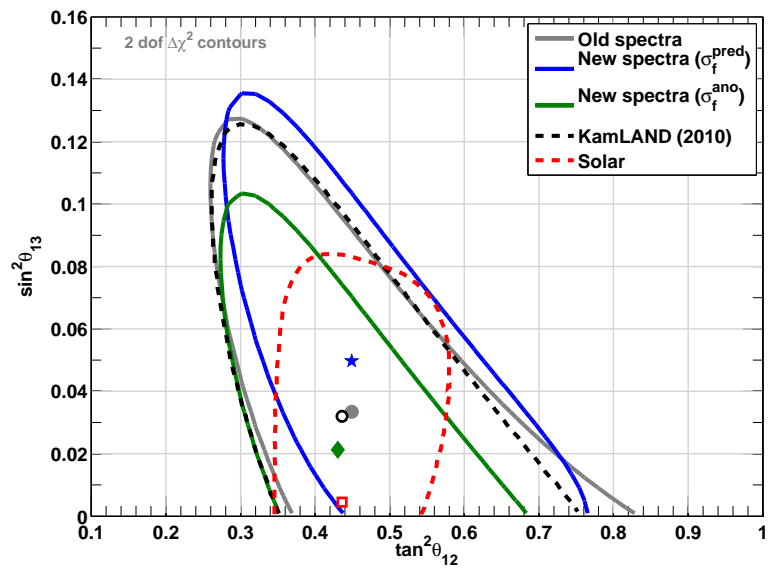

FIG. 10. Our simulation (gray line, gray disk) reproduces the published $95 \%$ C.L. KamLAND inclusion contour [17] represented by the black dashed line (black circle). We obtain the blue contour (star) by normalizing the expected no-oscillation antineutrino rate to $\sigma_{f}^{\text {pred,new }}$ and analyzing the data using the three active neutrino oscillations scheme. We then normalize the expected antineutrino event rate using $\sigma_{f}^{\text {ano }}$ to account for possible neutrino oscillations at very short baselines, leading to the green contour (diamond). This provides our new constraints in the $\tan ^{2} \theta_{12}-\sin ^{2} \theta_{13}$ plane, accounting for the reactor antineutrino anomaly. We note that the slight tension between the $\sin ^{2} \theta_{13}$ best-fit values of solar (red square) and reactor data is reduced in our scenario.

trino oscillation framework, the whole effect induced by the normalization shift is absorbed by the new oscillation at very short baselines, driven by $\Delta m_{\text {new }}^{2}$. Normalizing KamLAND's data to $\sigma_{f}^{\text {ano }}$ leads to an estimator revising the constraint on $\theta_{13}$ using the three-active neutrino oscillation framework, such that $\sin ^{2}\left(2 \theta_{13}\right)<0.31$ (1 dof) at $90 \%$ C.L. for $\Delta m_{31}^{2}=2.410^{-3} \mathrm{eV}^{2}$.

\section{Combining CHOOZ and KamLAND}

In sections $\mathrm{VIA}$ and $\mathrm{VIB}$ we separately revisited the CHOOZ and KamLAND results. Here, we first voluntarily ignore the reactor antineutrino anomaly at short baselines. We thus normalize both CHOOZ and KamLAND with $\sigma_{f}^{\text {pred,new }}$. Combining these two results leads to a best-fit value of $\sin ^{2}\left(2 \theta_{13}\right)=0.13_{-0.06}^{+0.06}$, barely consistent with a null value of $\theta_{13}$. The left panel of Figure 11 shows the $\Delta \chi^{2}$ profiles for CHOOZ (green), KamLAND (blue), and for their combination (red), as a function of $\sin ^{2}\left(2 \theta_{13}\right)$, marginalizing over the other parameters. In this scenario the revised limit is $\sin ^{2}\left(2 \theta_{13}\right)<0.23$ at $90 \%$ C.L. (1 dof), for the estimation of $\theta_{13}$ only, for $\Delta m_{31}^{2}=2.410^{-3} \mathrm{eV}^{2}$. These results are consistent with analyses of various data sets indicating a 'hint' for a nonzero $\theta_{13}[18,19]$. This is explained by the increase of the predicted event rate at $\mathrm{CHOOZ}$ and KamLAND, which

\begin{tabular}{c|c|c|c|c}
\hline \hline & \multicolumn{2}{|c|}{$\sigma_{f}^{\text {pred,new }}$} & \multicolumn{2}{c}{$\sigma_{f}^{\text {ano }}$} \\
\hline Re-analysis & Best fit & & $90 \%$ C.L. & Best fit \\
\hline$-1 \sigma$ & 90 & $90 \%$ C.L. \\
\hline KamLAND & $0.20_{-0.14}^{+0.13}$ & $<0.41$ & $0.09_{-0.14}^{+0.14}$ & $<0.31$ \\
CHOOZ & $0.11_{-0.08}^{+0.07}$ & $<0.22$ & $-0.02_{-0.08}^{+0.08}$ & $<0.10$ \\
Combined & $0.13_{-0.06}^{+0.06}$ & $<0.23$ & $0.00_{-0.07}^{+0.07}$ & $<0.11$ \\
\hline \hline
\end{tabular}

TABLE IV. Best-fit values for $\sin ^{2}\left(2 \theta_{13}\right)$ and $1 \sigma$ errors, considering two possible normalizations with $\sigma_{f}^{\text {pred,new }}$ or $\sigma_{f}^{\text {ano }}$. Limits are given for a 1 dof parameter estimation.

is attributed to $\theta_{13}$ only, in the three neutrino oscillations framework.

But the previous estimation does not take into account the reactor antineutrino anomaly. Let us now consider that both CHOOZ and KamLAND are normalized with $\sigma_{f}^{\text {ano }}$, in order to constrain $\theta_{13}$ using a three active neutrino oscillation framework. The right panel of Figure 11 shows the $\Delta \chi^{2}$ profiles for CHOOZ (green), KamLAND (blue), and for their combination (red), computed as before. In this scenario the best fit is at $\sin ^{2}\left(2 \theta_{13}\right)=0.0_{-0.07}^{+0.07}$. This lower best fit value is due to the fact that we predict fewer antineutrinos at CHOOZ and KamLAND, some of them having already oscillated into non-standard neutrinos. This leads to our revised constraint, $\sin ^{2}\left(2 \theta_{13}\right)<0.11$ at $90 \%$ C.L. (1 dof) for $\Delta m_{31}^{2}=2.410^{-3} \mathrm{eV}^{2}$. Results are summarized in Table IV.

We conclude that the hint for a non zero value of $\theta_{13}$, first presented in Ref. [18], vanishes if one normalizes CHOOZ's and KamLAND's predicted antineutrino rates to $\sigma_{f}^{\text {ano }}$. Furthermore we notice that the slight tension affecting the $\sin ^{2} \theta_{13}$ best-fit values of solar and reactor data [19] is reduced in our scenario involving a nonstandard neutrino such that $\Delta m_{\text {new }}^{2} \gg \Delta m_{31}^{2}$.

A full analysis of the data based on non-standard neutrino oscillation models would lead to more accurate results, beyond the scope of this article.

\section{Daya Bay, Double Chooz, and RENO}

Three new reactor neutrino oscillation experiments, Daya Bay [14], Double Chooz [15], and RENO [16] will soon be coming online to significantly improve our knowledge of reactor antineutrino rates at various baselines.

Among these experiments Double Chooz is the only one to operate a first phase without a near detector. The evidence for a reactor antineutrino anomaly triggers the discussion of the choice of the cross section per fission to be used for constraining $\theta_{13}, \sigma_{f}^{\text {pred,new }}$ or $\sigma_{f}^{\text {ano }}$, as well as its associated uncertainty.

Straightforwardly using $\sigma_{f}^{\text {pred,new }}$ in the three neutrino oscillation framework to interpret a hypothetical antineu- 

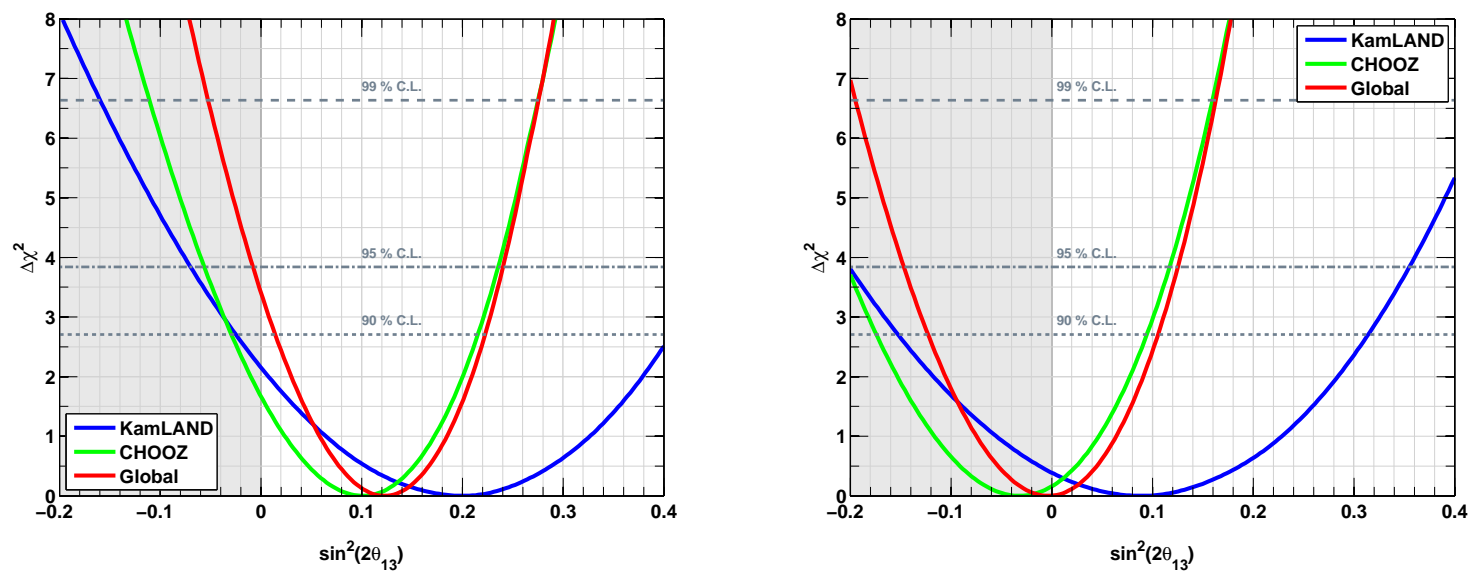

FIG. 11. Dependence of $\Delta \chi^{2}$ on $\sin ^{2}\left(2 \theta_{13}\right)$ for CHOOZ (green), KamLAND (blue), and their combination (red). Comparing the data with the excess in expected event rate predicted using $\sigma_{f}^{\text {pred,new }}$ leads to an increase of the best-fit value of $\sin ^{2}\left(2 \theta_{13}\right)$, in the three active neutrino oscillations framework (left). The normalization of the expected event rate with $\sigma_{f}^{\text {ano }}$ allows to absorb the reactor antineutrino anomaly observed at very short baseline, leading to our revised $\operatorname{constraints}$ on $\sin ^{2}\left(2 \theta_{13}\right)$.

trino deficit at Double Chooz could lead to an overestimation of $\theta_{13}$, which could fake a discovery. But if the sterile neutrino oscillation hypothesis discussed in this paper is proven, the use of $\sigma_{f}^{\text {pred,new }}$ would be possible in active+sterile neutrino oscillation frameworks. In that case the $2.7 \%$ error budget of the antineutrino spectra should be used. Assuming that the average experimental results of reactor neutrino are correct, using $\sigma_{f}^{\text {ano }}$ has the advantage to absorb either a antineutrino flux miscalculation, or a physical neutrino deficit at very short baselines, leading to a conservative constraint on $\theta_{13}$. The error budget could then be taken as the weighted standard deviation of the short baseline experiments, $1.0 \%$.

This choice is however not relevant for experiments running with a multi-detector configuration, since they absorb part of the uncertainty of the reactor antineutrino fluxes, depending on the setup. Even in the hypothetical case of antineutrino oscillation at very short baselines the sensitivities of Daya Bay, Double Chooz, and RENO should be marginally affected because of the large allowed values of $\Delta m_{\text {new }}^{2}$. However this statement should be checked based on detailed simulations.

In the non-standard neutrino hypothesis the discovery of a shape distortion in the positron energy spectrum of the far detectors may be determinant in disentangling $\theta_{13}$ from $\theta_{\text {new }}$.

\section{TESTING THE ANOMALY}

\section{A. At reactors}

The presence of sterile neutrinos would leave its imprint on the signal at both the near and far detectors of forthcoming reactor neutrino experiments. Taking re- sults from multi-detectors and allowing for the possibility of sterile mixing angles, one can probe both $\theta_{13}$ and the sterile mixing angle $\theta_{\text {new }}$ at Daya Bay, Double Chooz, and RENO [50]. Positron spectrum energy distortions should be deeply investigated at near detectors, as quoted in 52]. In any case, measurements of the expected over predicted event rates at the near detectors will allow the probing of the reactor antineutrino anomaly, providing high statistics and high precision measurements at a few hundreds meters from the antineutrino sources. The antineutrino anomaly would be best tested by performing blind analyses for all near detector data.

Further measurements at very short baselines below 100 meters would be useful to confirm the $\mathrm{MeV}$ electron antineutrino deficit. Currently no fundamental research program is underway to search for new oscillation physics at reactors. However there is a worldwide program at short baselines for the purpose of nuclear non-proliferation, using antineutrinos as new IAEA safeguards tools 53]. In this context the Nucifer experiment, located 7 meters away from the Osiris research reactor core in Saclay, will start its operation in 2011 [54]. Nucifer will thus have the possibility to test the anomaly. A rate-only analysis with a precision of a few percent may not be enough to provide a decisive improvement of the understanding of the anomaly. But a shape analysis may provide enough information, depending of the energy resolution.

We note here that the antineutrino non-proliferation program will not be affected by the antineutrino anomaly since relative antineutrino rates with respect to known thermal powers could be used to calibrate the experiments. 


\section{B. MegaCurie radioactive sources}

As mentioned above, radiochemical gallium experiments (Gallex and Sage) tested their experimental procedure by exposing their gallium target to MegaCurie neutrino sources using ${ }^{51} \mathrm{Cr}$ or ${ }^{37} \mathrm{Ar}$ [57]. The production and handling of such devices is thus well under control. Ref. 64] proposed to use a ${ }^{51} \mathrm{Cr}$ source inside two concentric gallium tanks, whereas Ref. [65] proposed to use a ${ }^{51} \mathrm{Cr}$ or ${ }^{90} \mathrm{Sr}$ source next to the Borexino detector. In liquid scintillating detectors ${ }^{51} \mathrm{Cr}$ or ${ }^{37} \mathrm{Ar} \nu_{e}$ 's are detected through neutrino-electron elastic scattering while ${ }^{90} \mathrm{Sr} \bar{\nu}_{e}$ 's are detected through inverse beta decay. With the $\Delta m_{\text {new }}^{2}$ values best fitting the sterile neutrino hypothesis, the deployment of a radioactive source at the center of an ultra-low background neutrino detector, such as Borexino, KamLAND, and SNO+, would allow both the testing of the $\nu_{e}$ deficit and the search for an oscillation pattern as function of the detector radius. These neutrino sources emit quasi-mono-energetic neutrino lines of sub-MeV energies leading to a clear oscillation pattern at the range of a meter. In addition a ${ }^{37} \mathrm{Ar}$ source emits only $\gamma$-rays through second order processes and is therefore easy to handle after its irradiation inside a breeder nuclear reactor. As an example, if a source of $1 \mathrm{MCi}$ were inserted in the middle of a large detector like $\mathrm{SNO}+$, it would provide a few hundred thousand interactions in the detector, of which a few ten thousands would deposit more than $500 \mathrm{keV}$. A $<15 \mathrm{~cm}$ accurate vertex reconstruction could allow to draw a simple and clear figure of the number of neutrino interactions as a function of radius, directly testing the sterile neutrino oscillation pattern for $\Delta m_{\text {new }}^{2}<5 \mathrm{eV}^{2}$.

\section{CONCLUSIONS AND OUTLOOK}

The impact of the new spectra of reactor antineutrino is extensively studied in this article. The increase of the expected antineutrino rate by about $3.5 \%$ combined with revised values of the antineutrino cross section significantly decreased the normalized ratio of observed to expected event rates in all previous reactor experiments performed over the last 30 years at distances below $100 \mathrm{~m} \mathrm{[2} \mathrm{9,} \mathrm{13,} \mathrm{44].} \mathrm{The} \mathrm{average} \mathrm{ratio} \mathrm{is} 0.943 \pm 0.023$, leading to the reactor antineutrino anomaly. This deficit could still be due to some unknown in the reactor physics, but we also analyze these revised results in terms of a suppression of the $\bar{\nu}_{e}$ rate at short distance as could be expected from a sterile neutrino, beyond the standard model, with a large $\left|\Delta m_{\text {new }}^{2}\right| \gg\left|\Delta m_{31}^{2}\right|$. We note that hints of such results were already present at the ILL neutrino experiment in 1981 [36]. We also considered that other neutrino experiments, MiniBooNE [59] and the gallium neutrino sources experiments [57], observe $\nu_{e}$ deficits at a similar $L / E$. These anomalies were comprehensively studied in Ref. [56]. It is important to note that these anomalies exist and are com- parable in both the neutrino and the antineutrino sectors. Furthermore it turns out that each experiment fitted separately leads to similar values of $\sin ^{2}\left(2 \theta_{\text {new }}\right)$ and similar lower bounds for $\left|\Delta m_{\text {new }}^{2}\right|$, but without a strong significance. Hence, we combined in a global fit these results with our re-evaluation of the reactor experimental results, taking into account the existing correlations. This leads to a solution for a new neutrino oscillation, such that $\left|\Delta m_{\text {new }}^{2}\right|>1.5 \mathrm{eV}^{2}$ (95\% C.L.) and $\sin ^{2}\left(2 \theta_{\text {new }}\right)=0.14 \pm 0.08$ (95\% C.L.), disfavoring the nooscillation case at $99.8 \%$ C.L. This hypothesis should be checked against systematical effects, either in the prediction of the reactor antineutrino spectra or in the experimental results.

We then revised the constraints on the $\theta_{13}$ mixing angle obtained with the three active neutrino oscillation framework by CHOOZ [13] and KamLAND [17]. We show that a $\bar{\nu}_{e}$ deficit measured at any antineutrino experiment with a baseline greater than $1 \mathrm{~km}$ could be misinterpreted as a hint for a non-zero value of $\theta_{13}$. Accounting for a possible $\left|\Delta m_{\text {new }}^{2}\right|$-driven sterile neutrino oscillation, we revise the constraint on the third mixing angle, such that $\sin ^{2}\left(2 \theta_{13}\right)<0.11$. Note that the KamLAND best fit of the solar neutrino parameters are unchanged. However, the combination of KamLAND and CHOOZ leads to a best fit value of $\sin ^{2}\left(2 \theta_{13}\right)=0.0_{-0.07}^{+0.07}$, in good agreement with the best fit value extracted from the combined analysis of solar neutrino data. This relaxes the tension between reactor and solar data recently reviewed in Ref. [19].

If the existence of a fourth neutrino turns out to be verified we note that a $7 \%$ reduction of the total flux of solar neutrinos must be taken into account when confronting with the experimental results. In particular the total neutrino $\nu$ flux measured by SNO [68], at $5.14 \pm 0.2110^{6} \mathrm{~cm}^{-2} \cdot \mathrm{s}^{-1}$, is now in better agreement with the prediction of the (reduced) high-Z Sun Standard Model model, at $5.25 \pm 0.910^{6} \mathrm{~cm}^{-2} . \mathrm{s}^{-1}$, and disfavors the (reduced) low-Z one $\left(4.09 \pm 0.910^{6} \mathrm{~cm}^{-2} . \mathrm{s}^{-1}\right)$. We note that the high- $Z$ model is also in good agreement with the data from helioseismology, contrarily to the low-Z model [69].

Assuming a hypothetical new neutrino $\nu_{\text {new }}$ heavier than the three active neutrinos we can briefly quantify its contribution to the effective masses searched for in $\beta$-decay and neutrino-less $\beta \beta$-decay experiments, as performed in Ref. [56]. The Tritium $\beta$-decay experiments have reported $m_{\beta} \lesssim 2 \mathrm{eV}(95 \%)$ [67]. A fourth neutrino with a mass in the $\mathrm{eV}$ range should contribute more than the active neutrinos, which are expected to have sub$\mathrm{eV}$ masses, to the signal searched for in direct detection beta decay experiments. We find that the contribution of the fourth neutrino state fitting the data analyzed in this article would contribute to $m_{\beta}$ for more than $0.2 \mathrm{eV}$ (95\% C.L.). This is within the sensitivity range of the forthcoming KATRIN experiment [70]. Assuming Majorana neutrinos, the contribution of the fourth state would be such that $m_{\beta \beta} \gtrsim 0.02 \mathrm{eV}$ (95\% C.L.), which is above 
the contribution of the three active neutrinos and thus disfavors possible cancellations with the three other active flavors [56, 71]. This contribution is of interest for the forthcoming experiments 72 .

Furthermore we would like to stress that the existence of a fourth neutrino is slightly favored by some recent cosmological data. The effective number of neutrino species fitted by WMAP and BAO observations 73. is $\mathrm{N}_{\text {eff }}=4.34 \pm 0.87$. An analysis of WMAP combined with the Atacama Cosmology Telescope data [74] leads to $\mathrm{N}_{\text {eff }}=5.3 \pm 1.3$, and an independent analysis of the WMAP 7 years data [75] provides $\mathrm{N}_{\text {eff }}=4 \pm 1$. Finally a recent analysis based on non-standard big bang nucleosynthesis [76] leads to $\mathrm{N}_{\text {eff }}=3.78 \pm 0.75$. But the compatibility of the sterile neutrino hypothesis exposed in this paper $\left(\left|\Delta m_{\text {new }}^{2}\right|>1.5 \mathrm{eV}^{2}, \sin ^{2}\left(2 \theta_{\text {new }}\right)=0.14 \pm 0.08\right.$ at $95 \%$ C.L.) with cosmological models and data should be assessed, especially its contribution to the nonbaryonic dark matter of the Universe, which may be non negligible.

Finally, a clear experimental proof of the presence of this fourth non-standard neutrino becomes mandatory. This can be given by the imprint on the energy spectrum in a very short baseline reactor neutrino experiment or by a new neutrino source experiment in a detector with energy and spatial resolution.

\section{ACKNOWLEDGMENTS}

We would like to thank M. Fallot, J. Gaffiot, L. Giot, J. Martino, A. Porta, V. Rulhmann-Kleider, J.-L. Sida, and F. Yermia for their careful reading of this manuscript. We are grateful to H. de Kerret, J. Rich, B. Mansoulié and S. Schoenert for discussions and suggestions. We thank J. Beacom and P. Vogel for fruitful exchange concerning the precise evaluation of the V-A inverse beta decay cross section.

\section{Appendix A: Error propagation in reactor experiment analyses}

One of the main difficulties in these calculations is the propagation of the errors on the ILL data from 22 24 to the final result, i.e. the cross-section per fission for each of the isotopes. From our previous work in [20] we propagated the error on the ILL data and the conversion procedure to the resulting neutrino spectra. However the binning and the energy range are then those of the ILL data $\left(250 \mathrm{keV}\right.$ bins from 2 to $8 \mathrm{MeV}$ for ${ }^{235} \mathrm{U}$ ).

These data are then fitted to an exponentialpolynomial model, see for example [47]. This allows to use arbitrarily fine binning, which is necessary for the correct convolution with the inverse beta cross-section and also the oscillation probability. In [47] the authors outline a method to propagate the errors and correlations on the polynomial coefficients to the physical observable.
However as they point out the correlation matrices obtained from the fit are rather unstable, most coefficients being strongly correlated or anti-correlated to each other. We found that this made error propagation very difficult.

As an exercise, we performed the following simple Monte-Carlo simulation: using the original correlation matrix on the converted ILL spectra, we simulated a series of ILL converted spectra, and fitted each of them using the exponential-polynomial model from [47]. We then evaluated the fitted polynomials in each energy bin, yielding fitted Monte-Carlo neutrino spectra. We then computed the bin-to-bin correlation matrix of these MonteCarlo spectra.

An example of the result is shown in Figure 13, along with the original correlation matrix in Figure12, Clearly the polynomial fit induces long-range correlations and anti-correlations between spectrum bins, and washes out the original correlations. We concluded that while we could rely on the polynomial fit for the mean values of the spectra (since the fit residuals are acceptably small), we could not use the resulting correlation matrices for error propagation.

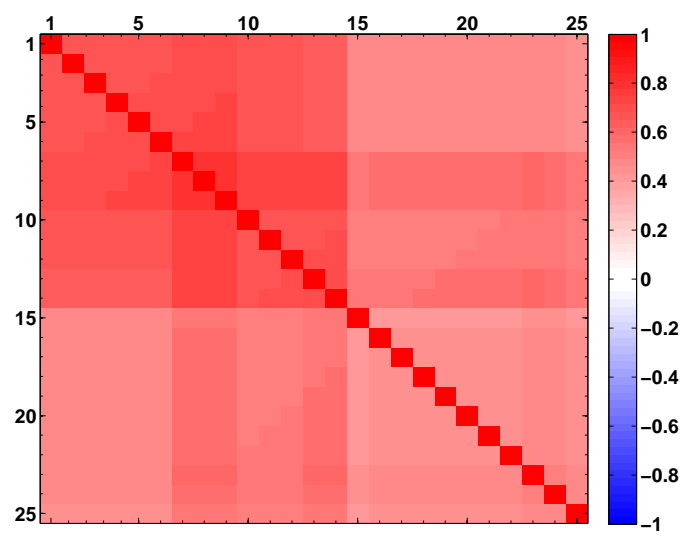

FIG. 12. Bin-to-bin correlation matrix $(25 \times 25$ bins, $2-8 \mathrm{MeV})$ of converted neutrino spectrum from ${ }^{235} \mathrm{U}$, including the ILL experimental errors and conversion effects from [20].

We therefore choose to propagate the original correlation matrix on our final binning by interpolating linearly between each bin of the original covariance matrix, and then computing the resulting correlation matrix. For bins which were not present in the original energy range, we take a constant error of $20 \%$, fully correlated bin-to-bin in order to obtain a conservative upper bound on the error. This allows us to use any binning over any energy range. Of course the resulting matrix still has rank 25 over the 2-8 MeV range, but since we do not need its inverse this does not limit our ability to use it for error propagation. An example of the resulting matrix can be found in Figure 14 for ${ }^{235} \mathrm{U}$.

Finally, as a cross-check of this method, we computed the error on the cross-section per fission for each isotope, 


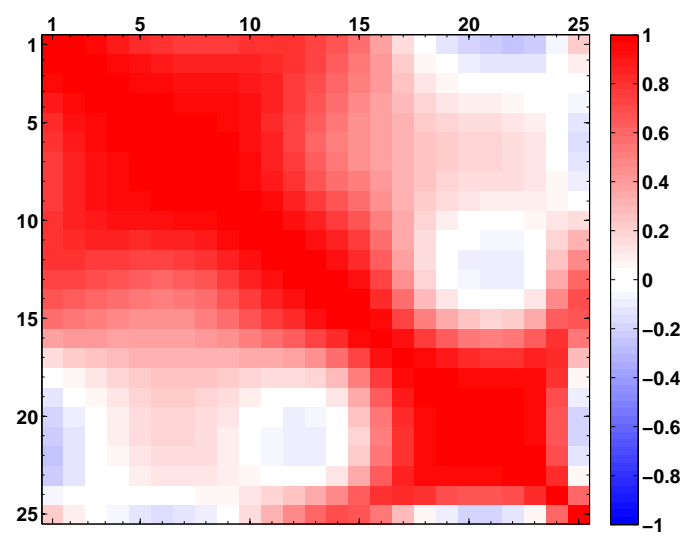

FIG. 13. Bin-to-bin correlation matrix $(25 \times 25$ bins, 2$8 \mathrm{MeV}$ ) of fitted neutrino spectrum from ${ }^{235} \mathrm{U}$, following the toy Monte-Carlo procedure outlined in the text. While the structure of Figure 12 is still present it has been significantly washed out and anti-correlation areas have appeared.

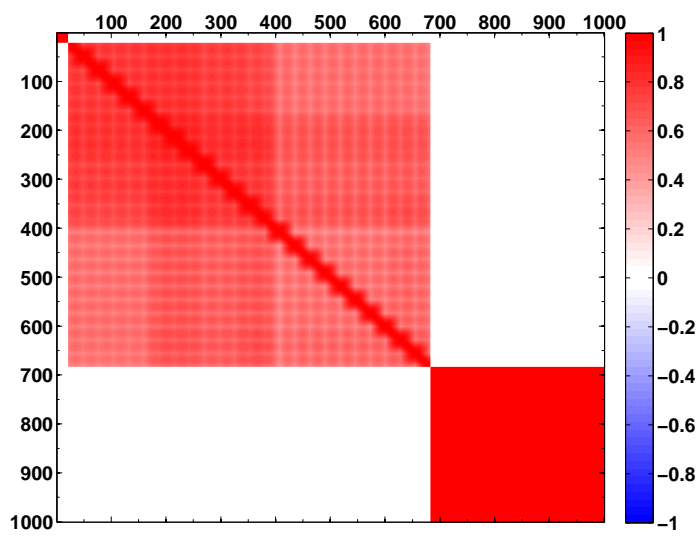

FIG. 14. Bin-to-bin correlation matrix $(1000 \times 1000$ bins, 1.75-10 MeV) of the fitted neutrino spectrum from ${ }^{235} \mathrm{U}$, obtained after linear interpolation of the original matrix, and extrapolating to a fully correlated $20 \%$ error outside the original binning. Clearly the experimental correlations from the ILL measurements are kept with this technique.

using our new error propagation method with interpolated ILL and conversion correlation matrices (column 2 of table I reproduced in table $\mathrm{V}$ below), and directly folding the converted ILL spectra with the inverse beta decay cross-section. Clearly, the results are extremely close. The effect of the error on the extrapolated part of the spectrum is found to be negligible. We also verified that the resulting error bars do not significantly depend on the chosen binning. These calculations validate the use of the exponential-polynomial fit along with the interpolation technique for error propagation on the final

\begin{tabular}{c|c|c}
\hline \hline & This work & ILL spectra \\
Isotope & 1000 bins, $1.8-10 \mathrm{MeV}$ & 25 bins, 2-8 MeV \\
\hline$\sigma_{f, 235 \mathrm{U}}^{\text {pred }}$ & $6.61 \pm 2.11 \%$ & $6.61 \pm 2.13 \%$ \\
$\sigma_{f, 239}^{\text {pred }}$ & $4.34 \pm 2.45 \%$ & $4.33 \pm 2.46 \%$ \\
$\sigma_{f, 241 \mathrm{Pu}}^{\text {pred }}$ & $5.97 \pm 2.15 \%$ & $6.02 \pm 2.16 \%$ \\
\hline \hline
\end{tabular}

TABLE V. Comparison between cross-sections per fission obtained from fitted spectra (central column), and from a direct convolution of the converted ILL spectra (right column). The error bars are 'exact' in the case of the direct convolution, while they are approximate for the fitted spectrum, obtained from interpolation of the original matrices.

spectrum, and allow us to use the binning of our choice without affecting the errors.

For ${ }^{238} \mathrm{U}$, since the spectrum is obtained from ab initio calculations, each bin is given an error ranging from $10 \%$ at low energy to $20 \%$ at high energy. However, if these errors are uncorrelated, the uncertainty on the overall neutrino rate and hence the cross-section per fission for ${ }^{238} \mathrm{U}$ is artificially low. To avoid this, we consider that there is a fully correlated $10 \%$ error on bins from 2 to $5.5 \mathrm{MeV}$, another fully correlated $15 \%$ error on the block from 5.5 to $6.75 \mathrm{MeV}$ and another $20 \%$ fully correlated error on the last bins of the spectrum. With this conservative prescription our error on the ${ }^{238} \mathrm{U}$ cross-section per fission is $8.2 \%$.

Finally we derive the total error, $\sigma_{S_{\text {tot }}}$, on the antineutrino rate, for a given core composition. We account for the uncertainties on the cross section per fission per isotope, and the uncertainties on the averaged fraction of fission per isotopes. As an example we can consider the case of Bugey-4. The fuel composition is given in Ref. [3]. The new values of the cross sections per fission per isotope as well as their uncertainties are given in Table [I The $f_{k}$ coefficients are taken as correlated, following a typical fuel evolution curve, and such that $\sum_{k} f_{k}=1 \pm 0.6 \%$ to account for the error on the thermal power of the nuclear core. In a first case we consider a $10 \%$ relative uncertainty on the fuel composition. We obtain a final error on the expected number of events of $2.7 \%$. Reducing the error on the fuel composition to $3.5 \%$ results in a final error of $2.4 \%$.

\section{Appendix B: Gallex/Sage, and MiniBooNE reanalysis}

In this appendix we briefly provide details on our reanalysis of published data from Gallex ${ }^{51} \mathrm{Cr}$ source data 62, 63], SAGE ${ }^{51} \mathrm{Cr}$ [60] and ${ }^{37} \mathrm{Ar}$ source data [61], and MiniBooNE neutrino data [59].

The Gallex collaboration performed two measurements with two ${ }^{51} \mathrm{Cr}$ sources in 62], obtaining the ratio of the measured and expected event rates. These data were recently reanalyzed [63], providing updated values of the 

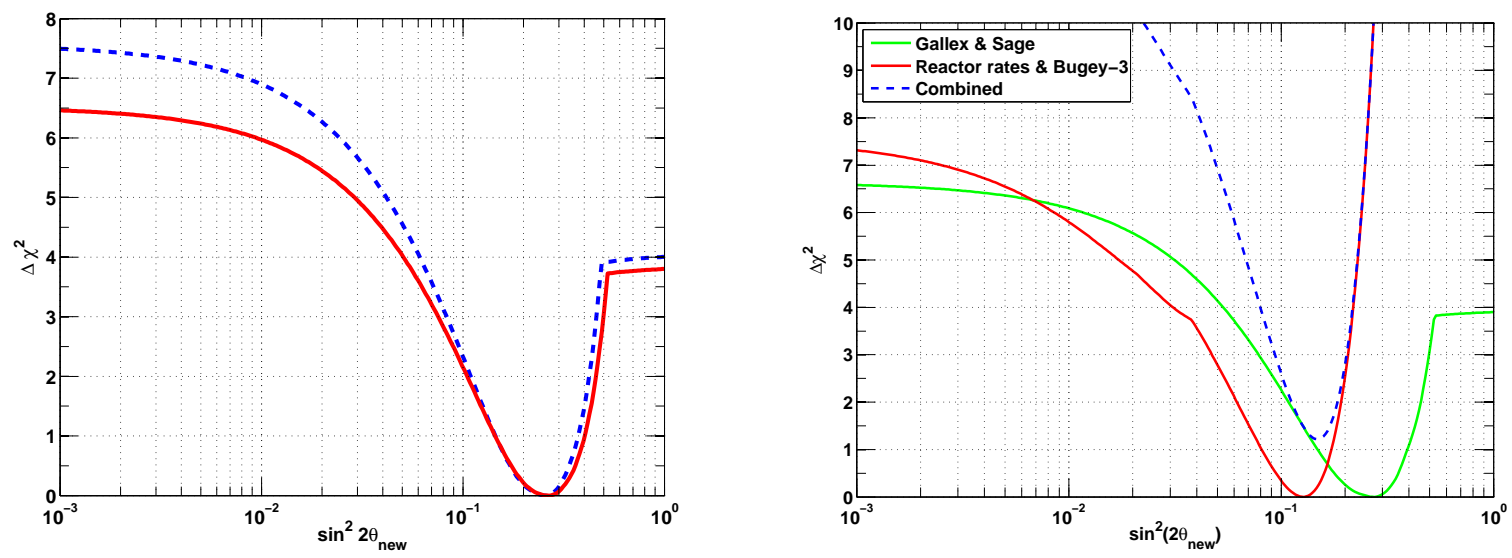

FIG. 15. (left) Reanalysis of the gallium calibration data of Gallex and Sage. $\Delta \chi^{2}$ projections as a function of $\sin ^{2}(2 \theta)$, without correlations (dashed) and with correlations as explained in the text. The dashed contour is very close to that of [56], validating this approach. (right) $\Delta \chi^{2}$ valleys of the $\sin ^{2}\left(2 \theta_{\text {new }}\right)$ parameter of the reactor antineutrino experimental results compared with the gallium results. Using the prescription of Ref. [77] the parameter goodness-of-fit is $27.0 \%$.

errors and the ratios. The SAGE collaboration performed a similar measurement with a ${ }^{51} \mathrm{Cr}$ source, and more recently with a ${ }^{37} \mathrm{Ar}$ source [60,61]. An analysis of these data in terms of neutrino oscillation was performed in [56, 58]. The data values are $0.95 \pm 0.11,0.81 \pm 0.11$, $0.95 \pm 0.12$ and $0.79 \pm 0.09$. Performing a neutrino oscillation search with these values yields contours very close to those of [56]. However we decided to include possible correlations between these four measurements in this present work, that were not previously taken into account. As the two Gallex measurements used the same experimental technique, we decided to fully correlate their systematic errors, which we understand to be $5.6 \%$ and $7.4 \%$ respectively. The statistical errors remain of course uncorrelated. For SAGE we followed a similar procedure, with systematics of $5.7 \%$ and $7.0 \%$ according to 60,61 .

Our fractional covariance matrix is

$$
\left(\begin{array}{cccc}
1.31 & 0.41 & 0 & 0 \\
0.41 & 1.55 & 0 & 0 \\
0 & 0 & 1.53 & 0.40 \\
0 & 0 & 0.40 & 1.30
\end{array}\right) 10^{-2}
$$

As expected we obtained slightly more conservative contours than those of [56] with this method.

It turns out that the $\sin ^{2}\left(2 \theta_{\text {new }}\right)$ best fits obtained from reactor antineutrino rate results only $\left(\sin ^{2}\left(2 \theta_{\text {new,bestfit }}\right) \sim 0.10\right)$ and the gallium results only $\left(\sin ^{2}\left(2 \theta_{\text {new, bestfit }}\right) \sim 0.27\right)$ are slightly different, though with large uncertainties. We quantified this effect by using the prescription of Ref. [77]. The corresponding $\Delta \chi^{2}$ valleys are displayed on the right panel of Figure 15. After marginalizing over $\left|\Delta m_{\text {new }}^{2}\right|$ (since fitted at the same value in both data sets) the parameter goodness-of-fit between the two data sets on $\sin ^{2}\left(2 \theta_{\text {new }}\right)$ is $27.0 \%$, indicating no significant tension between neutrino and antineutrino anomalies.

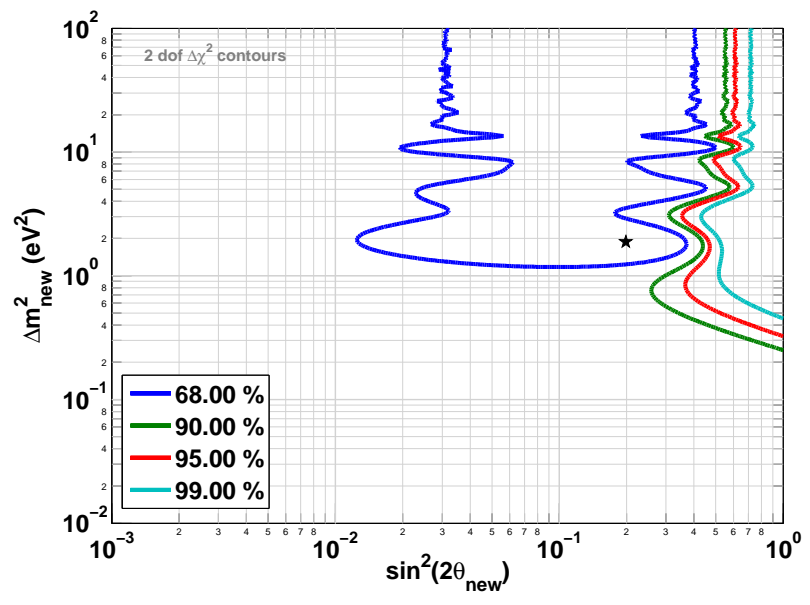

FIG. 16. Analysis of the MiniBOONE data following a procedure very similar to that of [56]. Our results are in good agreement.

We also analyzed the MiniBooNE neutrino data from [59] following the method outlined in [56]. The idea is to fit both electron-like and muon-like data at the same time. However, instead of searching for $\nu_{\mu} \rightarrow \nu_{e}$ appearance, we search for $\nu_{e}$ disappearance, while allowing the global normalization of all the samples (e-like and mulike) to fluctuate.

The excess of e-like data in the low energy part of the spectrum is well-fitted by the combined increase in overall normalization and by the disappearance of $\nu_{e}$ induced e-like events. We used a simplified version of the analysis in reference [56], in that we used a covariance matrix independent from the normalization fitting parameter $f$. We obtain very similar results, although our best fit point 
is in a slightly different location, at $\sin ^{2}(2 \theta)=0.23$, $\Delta m^{2}=1.88 \mathrm{eV}^{2}$ and $f=1.083$, as can be seen in Figure 16 .
We have only included this analysis of MiniBooNE's data for completeness. However in our final statistical significance it has very little impact.
[1] K. Nakamura et al., (Particle Data Group), J. Phys. G 37, 075021 (2010).

[2] H. Kwon et al., Phys. Rev. D24 1097 (1981).

[3] Y. Déclais et al., Phys. Lett. B338 (1994) 383.

[4] B. Achkar et al., Nucl. Phys. B434 (1995) 503.

[5] G. Zacek et al., Phys. Rev. D34 (1986) 2621.

[6] A.I. Afonin et al., JETP 94 (1988) 1-17.

[7] V. Kuvshinnikov et al., JETP 54 N5 (1991) 259.

[8] G.S. Vidyakin et al., JETP. 93 (1987) 424-431.

[9] G.S. Vidyakin et al., JETP Lett. 59 (1994) 390.

[10] C.L. Jr. Cowan, F. Reines, F.B. Harrison, H.W. Kruse and A.D. McGuire, Science 124 (1956) 103

[11] Z.D. Greenwood et al., Phys. Rev. D53 11 (1996).

[12] Th. Lasserre and H.W. Sobel., Comptes Rendus Physique, French Academy of Sciences, 6, 749-757 (2005) arXiv:nucl-ex/0601013

[13] M. Apollonio et al., Phys. Lett. B466, 415 (1999). M. Apollonio et al., Eur. Phys. J. C27, 331-374 (2003).

[14] X. Guo et al., arXiv:hep-ex/0701029.

[15] F. Ardellier et al., arXiv:hep-ex/0606025

[16] J. K. Ahn et al., arXiv:hep-ex/1003.1391.

[17] A. Gando et al., arXiv:1009.4771 v2.

[18] G. L. Fogli, E. Lisi, A. Marrone, A. Palazzo, A. M. Rotunno, Phys. Rev. Lett. 101, 141801 (2008).

[19] M. Mezzetto, T. Schwetz, J. Phys. G37, 103001 (2010).

[20] Th. A. Mueller, D. Lhuillier et al., arXiv:1101.2663 (submitted to PRC); Th. A. Mueller, PhD Thesis, Paris-Sud XI University, 2010.

[21] K. Schreckenbach et al., Phys. Lett. 99B, 251 (1981).

[22] K. Schreckenbach et al., Phys. Lett. 160B, 325 (1985).

[23] F. von Feilitzsch, A. A. Hahn and K. Schreckenbach, Phys. Lett. 118B, 162 (1982).

[24] A. A. Hahn et al., Phys. Lett. 218B, 365 (1989).

[25] B. R. Davis et al., Phys. Rev. C19, 2259 (1979). P. Vogel et al., Phys. Rev. C24, 1543 (1981).

[26] N. H. Haag, Bestimmung des Antineutrinospektrums der Spaltprodukte von ${ }^{238}$ U, Diplomarbeit, Technische Universität München.

[27] D. H. Wilkinson, Nucl. Phys. A 377, 474 (1982).

[28] D. H. Wilkinson, Z. Phys. A 348, 129 (1994).

[29] S. A. Fayans, Sov. J. Nucl. Phys. 42 (4), 590 (1985).

[30] P. Vogel and J. F. Beacom, Phys. Rev. D60 (4), 053003 (1999).

[31] C. Caso et al. (Particle Data Group), Eur. Phys. Jour. C3, (1999), 1.

[32] J. Liu et al., Phys. Rev. Lett. 105, 181803 (2010).

[33] H. Abele, Prog. Part. Nucl. Phys. 60 (2008) 1-81.

[34] A. P. Serebrov and A. K. Fomin, Phys. Rev. C82 (2010) 035501.

[35] A. Pilchmaier et al., Phys. Lett. B693 (2010) 221-226.

[36] A. Hoummada et al., Appl. Rad. Isot. Vol. 46, No. 6/7, pp. 449-450, (1995).

[37] D. Nicolo, Search for neutrino oscillations in a long baseline, experiment at the Chooz nuclear reactors, $\mathrm{PhD}$ (1999)

[38] P. Adamson et al., Phys. Rev. Lett. 101, 131802 (2008)
[39] P. Vogel, Phys. Rev. D29, 1918 (1984).

[40] P. Vogel et al., Phys. Rev. C24, 1543 (1981).

[41] V.I. Kopeikin, Preprint Kurchatov Institut of Atomic energy, Moscow IAE-4305/2, (1988).

[42] L.A. Mikaelyan, JETP Lett. 47, (1988).

[43] A.A. Kuvshinnikov et al., Sov. J. Nucl. Phys. 52, (1990) 300, JETP Lett. 54, (1991) 255.

[44] F. Boehm Phys. Rev. D64, 11 (2001).

[45] K. Schreckenbach et al., to be completed.

[46] http://www.iaea.org/programmes/a2/

[47] P. Huber and T. Schwetz, Phys. Rev. D70 (2004) 053011.

[48] G. Mention, T. Lasserre, and D. Motta, arXiv:0704.0498.

[49] J. J. Gomez-Cadenas and M. C. Gonzalez-Garcia, Z. Phys. C71, 443 (1996); S. Goswami, Phys. Rev. D55, 2931 (1997); S. M. Bilenky, C. Giunti and W. Grimus, Eur. Phys. J. C 1, 247 (1998).

[50] A. Bandyopadhyay, S. Choubey arXiv:0707.2481 1.

[51] M. Fallot et al., to be published.

[52] A. de Gouvea and T. Wytock arXiv:0809.5076

[53] IAEA Final Report: Focused Workshop on Antineutrino Detection for Safeguards Applications (2008)

[54] A. Porta et al., J. Phys. Conf. Ser. 203012092 (2010).

[55] C. Giunti, M. Laveder, Phys. Rev. D80 (2009) 013005.

[56] C. Giunti, M. Laveder, Phys. Rev. D82 (2010) 053005.

[57] P. Anselmann et al. (GALLEX), Phys. Lett. B342, 440 (1995); W. Hampel et al. (GALLEX), Phys. Lett. B420, 114 (1998); F. Kaether et al. Phys. Lett. B685, 47 (2010); J. N. Abdurashitov et al. (SAGE), Phys. Rev. Lett. 77, 4708 (1996); J. N. Abdurashitov et al. (SAGE), Phys. Rev. C59, 2246 (1999); J. N. Abdurashitov et al., Phys. Rev. C73, 045805 (2006); J. N. Abdurashitov et al. (SAGE), Phys. Rev. C80, 015807 (2009).

[58] C. Giunti, M. Laveder, arXiv:1006.3244v2.

[59] A. A. Aguilar-Arevalo et al., Phys. Rev. Lett. 98 (2007) 231801.; A. A. Aguilar-Arevalo et al., Phys. Rev. Lett. 102 (2009) 101802.

Data release available at www-boone.fnal.gov/

[60] J. N. Abdurashitov et al., Phys. Rev. C 59, 2246 (1999). arXiv:hep-ph/9803418

[61] J. N. Abdurashitov et al., Phys. Rev. C 73, 045805 (2006)

[62] W. Hampel et al., Phys. Lett. B 420, 114 (1998).

[63] F. Kaether, W. Hampel et al., Phys. Lett. B 685 (2010) 47-54.

[64] V. N. Gavrin, V. V. Gorbachev, E. P. Veretenkin, B. T. Cleveland arXiv:1006.2103

[65] A. Ianni , D. Montanino, G. Scioscia, Eur. Phys. J. C8 (1999) 609-617.

[66] F. Reines, H. W. Sobel, F. Pasierb, Phys. Rev. Lett. 45 (1980) 1307-1311. F. James, Lecture at the CERN School of Computing, CERN DD/81/02, 1980.

[67] C. Kraus et al., Eur. Phys. J. C40, 447 (2005); V. M. Lobashev, Nucl. Phys. A719, C153 (2003).

[68] B. Aharmim et al., The SNO Collaboration, Phys. Rev. C 81, 055504 (2010)

[69] C. PenaGaray, and A. Serenelli arXiv:0811.2424.

[70] KATRIN Collaboration, arXiv:hep-ex/0109033v1. 
[71] M. Maltoni, T. Schwetz, M. A. Tortola, J. W. F. Valle, New J. Phys. 6:122,2004.

[72] M. Pedretti et al., International Journal of Modern Physics A, 23 (2008) 3395; H. Simgen et al., Nucl. Phys. B143 (2005) 567; R. Arnold et al., Eur. Phys. J. CDOI 10.1140/epjc/s10052-010-1481-5 (2010).
[73] E. Komatsu et al., arxiv.org/abs/1001.4538.

[74] J. Dunkley et al., arxiv.org/abs/1009.0866.

[75] J. Hamann, S. Hannestad, G. G. Raffelt, I. Tamborra, and Y. Y.Y. Wong, arxiv.org/abs/1006.5276.

[76] Y. I. Izotov, T. X. Thuan, arxiv.org/abs/1001.4440.

[77] M. Maltoni, T. Schwetz, Phys. Rev. D68, 033020 (2003). 